\title{
Differential-Detection Aided Large-Scale Generalized Spatial Modulation Is Capable of Operating in High-Mobility Millimeter-Wave Channels
}

\author{
Naoki Ishikawa, Member, IEEE, Rakshith Rajashekar, Senior Member, IEEE, Chao Xu, Member, IEEE, \\ Mohammed El-Hajjar, Shinya Sugiura, Senior Member, IEEE, Lie-Liang Yang, and Lajos Hanzo, Fellow, IEEE
}

\begin{abstract}
A large-scale differential-detection aided generalized spatial modulation (GSM) system is proposed, which relies on a novel Gram-Schmidt basis set and an adaptive low-complexity detector, and is evidently suitable for high-mobility millimeter-wave (mmWave) channels. We consider non-stationary time-varying mmWave channels and assume that the beam-angles remain relatively fixed, while the channel coefficients vary rapidly. In this scenario, it is a challenging task to find the accurate estimates of channel coefficients for digital beamforming, which becomes an even more severe problem, as the numbers of subarrays and subcarriers increase. Our analog-beamforming-aided nonsquare differentially-detected scheme achieves a higher transmission rate than the conventional coherent multiple-input multiple-output schemes because the pilot overhead and the complex-valued feedback are eliminated. Our simulation results following the IEEE 802.11ad specifications show that the performance of our proposed nonsquare differential GSM improved upon increasing the number of subarrays, where the maximum transmission rate of $16[\mathrm{bps} / \mathrm{Hz}]$ was considered.
\end{abstract}

Index Terms-MIMO, OFDM, millimeter-wave communications, index modulation, spatial multiplexing, spatial modulation, differential modulation, differential spatial modulation, differential space-time block codes.

\section{INTRODUCTION}

G IVEN its ample bandwidth, millimeter-wave (mmWave) communications have the promise of a multi-Gbps throughput for supporting compelling wireless applications [14]. A large number of antenna elements are used to form a directed beam, in beamforming (BF), for mitigating the high propagation loss of mmWave carriers. Since the full-digital $\mathrm{BF}$ circuit is power-thirsty, the associated analog phase shifters are typically combined with digital precoding to simplify both

N. Ishikawa is with the Graduate School of Information Sciences, Hiroshima City University, Japan (e-mail: naoki@ishikawa.cc). R. Rajashekar, C. Xu, M. El-Hajjar, L. L. Yang and L. Hanzo are with the School of ECS, University of Southampton, UK (email: rmr1u14@soton.ac.uk, cx1g08@ecs.soton.ac.uk, meh@ecs.soton.ac.uk, 1ly@ecs.soton.ac.uk,1h@ecs.soton.ac.uk). S. Sugiura is with the Institute of Industrial Science, University of Tokyo, Meguro-ku, Tokyo 153-8505, Japan (sugiura@ieee.org).

The work of N. Ishikawa was partially supported by the Japan Society for the Promotion of Science (JSPS) KAKENHI (Grant Number 17H07036). The work of S. Sugiura was supported in part by the JSPS KAKENHI (Grant Number 16KK0120). The financial support of the EPSRC projects EP/P034284/1, EP/N004558/1 and EP/L018659/1, as well as of the Royal Society's GRCF Award and of the ERC's Advanced Fellow Award QuantCom is gratefully acknowledged. the transmitter and the receiver architectures [4-6]. Indoor mmWave communications in the unlicensed $57-66 \mathrm{GHz}$ band have been developed under the IEEE 802.11ad specifications for supporting up to $7 \mathrm{Gbps}$ throughput $[7,8]$, with the aid of orthogonal frequency division multiplexing (OFDM). Since 2017, the IEEE 802.11ay protocol set has been discussed, which relies on multiple-input multiple-output (MIMO) technologies [9] and aims for supporting a 100 Gbps throughput through eight independent data streams [10].

At the time of writing, index modulation has gathered tremendous attention [11-14], and it has also been applied to mmWave communications [15-30], because its unique on/off switching structure simplifies both the transmitter and the receiver implementation. Generalized spatial modulation (GSM) [31] activating more than one transmit subarrays has been used for indoor mmWave communications in order to obtain a sufficiently high BF gain [25-30]. Although having a low number of activated subarrays is inefficient in terms of BF, the GSM family is capable of achieving similar performance trends to the classic spatial multiplexing based MIMO family, such as Bell Laboratories layered space-time (BLAST) that activates all the transmit subarrays [13], despite having a low detection complexity.

In general, having a strong and stable line-of-sight (LoS) path is indispensable for high-rate mmWave communications. In LoS-dominant, large-scale and high-mobility scenarios the channel state information (CSI) has to be updated quite often and its increased feedback rate requires substantial computational resources $[32,33]$. In contrast to LoS-dominant scenarios, the channel coefficients may change rapidly [34-36] in non-LoS (NLoS) scenarios, while the corresponding direction angle is relatively fixed [37]. For example, when a few people walk at a fast pace in a room, the channel's coherence time is reduced compared to the slow-paced scenario [34]. Since the variance of path loss in NLoS scenarios is higher than that of LoS scenarios [35,36], the estimation of channel coefficients inevitably becomes frequent and time-consuming [37], which erodes the high rate of MIMO-aided mmWave communications.

To circumvent the channel-estimation overhead in sub$\mathrm{GHz}$ MIMO wireless communications, the differential spacetime coding (DSTC) philosophy has been proposed [38-41]. In particular, the sparse-matrix-based DSTC family [42-49] 
achieves a high reliability, despite its lower complexity than that of the classic dense-matrix-based scheme [38-40]. The sparse DSTC scheme is referred to as differential spatial modulation (DSM) [42], since it is a differential counterpart of coherent SM [50]. The DSM codeword has a sparse structure, hence it is capable of reducing the encoding and decoding complexities [13].

The DSM scheme that is capable of dispensing with the channel estimation overhead may be deemed attractive for high-rate high-mobility mmWave communications. However, the major impediment of the conventional DSM family is its performance loss in high-rate scenarios [13], which is imposed by having to satisfy the unitary constraint. The encoding and the decoding complexities inevitably escalate as the number of transmit antennas increases, even though the sparse structure may be exploited for reducing the complexity. This has been an open issue since 2000, and it was solved in [51,52] by invoking a simple square-to-nonsquare matrix mapping, which achieved a high throughput of $R=12$ [bps/Hz] for $M=1024$ transmit antennas. This high-rate capability was also verified by other authors in [53,54] based on [51].

Instead of the high-complexity square-matrix-based DSM, the low-complexity nonsquare-matrix-aided DSM scheme of [51-54] may be the best solution for high-rate mmWave communications. Nevertheless, the conventional nonsquare scheme of [51-54] requires a carefully designed forgetting factor relying on time-consuming optimization in a specific channel environment. Thus, it is a challenge for the conventional scheme to communicate over time-varying mmWave channels. Furthermore, the nonsquare-matrix-based scheme exhibits severe error floors in high-Doppler high-mobility scenarios [51, 52], which implies that the nonsquare concept is less attractive for realistic mobile wireless communications.

Against this background, we propose a high-rate nonsquare differential scheme for static and high-mobility mmWave communications, where a special form of the proposed scheme may be deemed equivalent to the differential counterpart of GSM. ${ }^{1}$ In high-mobility scenarios, we consider non-stationary and time-varying mmWave MIMO-OFDM channels, where it is a challenging task to accurately track the channel coefficients. The straightforward application of the conventional nonsquare concept [51,52] does not achieve a convincing performance gain in this scenario, due to the rapidly fluctuating channel coefficients and the fixed forgetting factor. To the best of our knowledge, the square and nonsquare DSTC family has not been applied to MIMO-aided mmWave communications because of its modest performance eroded by having to satisfy the unitary constraint and owing to the lack of channel coefficients for digital $\mathrm{BF}^{2}$ The major contributions of this paper are summarized as follows:

- We propose a novel basis set relying on the GramSchmidt process [56], which converts the conventional square and sparse DSM constellation into differential GSM. This proposed scheme activates an arbitrary num-

\footnotetext{
${ }^{1}$ Note that the conception of differential GSM was first heralded in [43].

${ }^{2}$ Note that the first analog-circuit-based mmWave broadcasting system in the 1980s adopted differential PSK [55].
}

ber of transmit subarrays and it is capable of striking a BF gain vs. detection complexity trade-off.

- We propose two adaptive forgetting factors for the proposed nonsquare DSTC, where both time-invariant and time-varying scenarios are considered. The proposed forgetting factor allows the detector to adaptively track the rapidly-fluctuating channel coefficients, hence improving the system's reliability.

- We demonstrate that the performance of our scheme monotonically improves upon increasing the number of subarrays and approaches that of its coherent counterpart having perfectly accurate channel coefficients, while the channel estimation overhead of the coherent MIMO escalates with the system's scale. When considering high-mobility scenarios, the proposed differential GSM scheme achieves a similar performance trend to that of its nonsquare counterpart that activating all the transmit subarrays, whilst reducing the detection complexity.

Furthermore, since the differential family has not been applied in MIMO-aided mmWave communications, we detail the main advantages of the proposed differential system as follows:

- Reduced feedbacks. Our system only requires realvalued signal-to-noise ratio (SNR) feedback for adjusting the beam angle, but does not require full CSI feedbacks. The CSI feedback contains a large number of complexvalued channel coefficients. Its frame length increases with the number of subarrays and subcarriers, as defined by the IEEE 802.11 specifications [57, p. 746], which reduces the effective throughput.

- No channel estimation overhead. Our scheme eliminates the pilot subcarriers, the interpolation-aided channel tracking, and the fixed-length channel estimation sequences, which increases the effective throughput. In the IEEE 802.11ad specifications, 16 pilot subcarriers are reserved for the entire set of 355 subcarriers [58], which represent about $5 \%$ of subcarriers. Here, the frequency domain channel transfer function coefficients are directly given for the pilot subcarriers, while for the data subcarriers they are estimated by interpolation [59-61]. Additionally, the IEEE 802.11ad frames include the fixedlength channel estimation field, whose duration is twice as long as the 512-subcarrier OFDM symbol duration [58].

- Adaptive operation. Our scheme subsumes the conventional DSTC family. In the IEEE 802.11ad specifications, single-input single-output (SISO) $\pi / 2$-differential binary phase-shift keying (DBPSK) is used before setting up the BF link [58], because DBPSK exhibits a high robustness against unexpected channel conditions. Our system can readily switch between the proposed and the conventional DPSK arrangement by simply changing the corresponding codebook.

- High BF gain. The conventional square-matrix-based single-active DSM scheme only achieves a limited analog $\mathrm{BF}(\mathrm{ABF})$ gain, because merely a single transmit subarray is activated [25]. By contrast, the proposed scheme activates multiple subarrays at the same time and thus 
it achieves a substantial $\mathrm{ABF}$ gain that is comparable to that of the conventional spatial multiplexing scheme such as BLAST.

The remainder of this paper if organized as follows. Section II defines the channel model considered in this paper. Section III reviews the conventional square- and nonsquarematrix-based DSTC family, while Section IV proposes our adaptive nonsquare differential GSM scheme. Section V provides our performance comparisons between the conventional and proposed schemes. Section VI concludes this paper.

We use the following notations in this paper. Italicized symbols represent scalar values, bold symbols represent vectors/matrices, and calligraphic symbols represent tensors. $\jmath=$ $\sqrt{-1}$ denotes the imaginary number, while $j$ denotes an index. $(\cdot)^{\mathrm{T}}$ denotes the transpose of a matrix, while $(\cdot)^{\mathrm{H}}$ denotes the Hermitian transpose of a matrix. Furthermore, $\mathcal{C N}\left(\mu, \sigma^{2}\right)$ denotes the complex normal distribution of a random variable having a mean of $\mu$ and a variance of $\sigma^{2}$. Finally, $\mathbb{C}^{A \times B \times C}$ represents a set of $(A \times B \times C)$-sized complex-valued tensors, $\mathbf{I}_{m}$ represents the $(m \times m)$ identity matrix, bdiag[.] constructs a block diagonal of its arguments, $\operatorname{diag}[\cdot]$ extracts the diagonal element of a matrix, and circulant $[\cdot]$ constructs a circulant matrix from a vector.

\section{TENSOR-BASED TIME-INVARIANT AND TIME-VARYING MMWAVE CHANNEL MODEL}

In this paper, we consider the popular clustered mmWave channel model of [37,62], where we have $N_{\mathrm{sc}}$ subcarriers, $M_{\mathrm{T}}$ transmit antenna (TA) elements, $N_{\mathrm{R}}$ receive antenna (RA) elements, and the $L$ multipath components. The TA elements are equally separated by half the wavelength, and are grouped into $M$ subarrays. Thus, each transmit subarray has $U_{\mathrm{T}}=M_{\mathrm{T}} / M$ TA elements. Similarly, the receiver has $N$ subarrays and each subarray has $U_{\mathrm{R}}=N_{\mathrm{R}} / N$ RA elements. Here, the direction angle is fixed and perfectly known at both the transmitter and the receiver, while the channel coefficients vary based on the autoregressive (AR) model designed for mmWave channels [63].

\section{A. Tensor-Based Modeling}

In this paper, we use a tensor-based formalism. A tensor is a multi-dimensional array [64]. We represent $L$ number of $N \times M$ channel matrices as a three-dimensional (3D) tensor $\mathcal{H}^{\mathrm{BF}} \in \mathbb{C}^{N \times M \times L}$ as follows: [37,62]

$\mathcal{H}_{:: l}^{\mathrm{BF}}=\frac{1}{\sqrt{L}} \underbrace{\mathbf{W}_{\mathrm{R}}^{\mathrm{H}}}_{\left(N \times N_{\mathrm{R}}\right)} \underbrace{\mathbf{a}_{\mathrm{R}}(l)}_{\left(N_{\mathrm{R}} \times 1\right)} \lambda(i, l) \underbrace{\mathbf{a}_{\mathrm{T}}(l)^{\mathrm{H}}}_{\left(1 \times N_{\mathrm{T}}\right)} \underbrace{\mathbf{W}_{\mathrm{T}}}_{\left(N_{\mathrm{T}} \times M\right)} \in \mathbb{C}^{N \times M}$,

where $\mathcal{H}_{n::}^{\mathrm{BF}} \in \mathbb{C}^{M \times L}(n=1, \cdots, N), \mathcal{H}_{: m:}^{\mathrm{BF}} \in \mathbb{C}^{N \times L}(m=$ $1, \cdots, M)$, and $\mathcal{H}_{:: l}^{\mathrm{BF}} \in \mathbb{C}^{N \times M}(l=1, \cdots, L)$ denote the two-dimensional (2D) sections of the 3D tensor $\mathcal{H}^{\mathrm{BF}}$. This notation is also detailed in [64]. Additionally, the integer $i$ denotes the transmission index for $i \geq 1$. In Eq. (1), $\lambda(i, l) \in$ $\mathbb{C}$ is generated for each $n$ and $m$. We use the following vector notation for simplifying the ABF weights:

$$
\mathbf{a}_{k}^{K}(\phi)=\left[\begin{array}{lll}
e^{j(k-1) \cos \phi} & \cdots & e^{j(k-K-1) \cos \phi}
\end{array}\right]^{\mathrm{T}} \in \mathbb{C}^{K},
$$

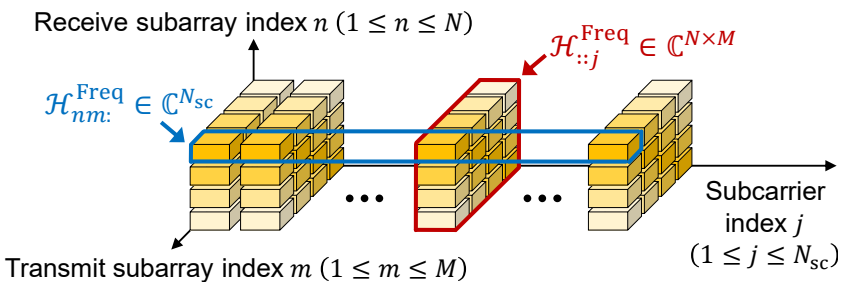

Fig. 1. The graphical overview of the 3D tensor $\mathcal{H}^{\text {Freq }} \in \mathbb{C}^{N \times M \times N_{\mathrm{sc}}}$.

which relies on a uniform linear array. In Eq. (1), $\mathbf{a}_{\mathrm{T}}(l)=$ $\mathbf{a}_{1}^{N_{\mathrm{T}}}\left(\phi_{\mathrm{T}}(l)\right) / \sqrt{N_{\mathrm{T}}}$ and $\mathbf{a}_{\mathrm{R}}(l)=\mathbf{a}_{1}^{N_{\mathrm{R}}}\left(\phi_{\mathrm{R}}(l)\right) / \sqrt{N_{\mathrm{R}}}$ denote the transmit and receive steering vectors associated with the $l$-th multipath component, where $\phi_{T}(l)$ and $\phi_{R}(l)$ represent the corresponding angle-of-departure (AoD) and angle-of-arrival (AoA), respectively. The antenna weight matrices $\mathbf{W}_{\mathrm{T}}$ and $\mathbf{W}_{\mathrm{R}}$ are constructed by [65]

$$
\begin{array}{r}
\mathbf{W}_{\mathrm{T}}=\frac{1}{\sqrt{U_{\mathrm{T}}}} \operatorname{bdiag}\left[\mathbf{a}_{1}^{U_{\mathrm{T}}}\left(\phi_{\mathrm{T}}^{\prime}(1)\right), \mathbf{a}_{U_{\mathrm{T}}+1}^{U_{\mathrm{T}}}\left(\phi_{\mathrm{T}}^{\prime}(2)\right),\right. \\
\left.\cdots, \mathbf{a}_{(M-1) U_{\mathrm{T}}+1}^{U_{\mathrm{T}}}\left(\phi_{\mathrm{T}}^{\prime}(M)\right)\right] \in \mathbb{C}^{N_{\mathrm{T}} \times M}
\end{array}
$$

and

$$
\begin{array}{r}
\mathbf{W}_{\mathrm{R}}=\frac{1}{\sqrt{U_{\mathrm{R}}}} \operatorname{bdiag}\left[\mathbf{a}_{1}^{U_{\mathrm{R}}}\left(\phi_{\mathrm{R}}^{\prime}(1)\right), \mathbf{a}_{U_{\mathrm{R}}+1}^{U_{\mathrm{R}}}\left(\phi_{\mathrm{R}}^{\prime}(2)\right),\right. \\
\left.\cdots, \mathbf{a}_{(N-1) U_{\mathrm{R}}+1}^{U_{\mathrm{R}}}\left(\phi_{\mathrm{R}}^{\prime}(N)\right)\right] \in \mathbb{C}^{N_{\mathrm{R}} \times N},
\end{array}
$$

where $\phi_{\mathrm{T}}^{\prime}(m)$ and $\phi_{\mathrm{R}}^{\prime}(n)$ are selected out of $L$ number of $\phi_{\mathrm{T}}(l)$ and $\phi_{\mathrm{R}}(l)$. Note that bdiag[.] represents the block diagonalization.

Next, we introduce a four-dimensional (4D) tensor $\mathcal{H}^{\text {Time }} \in$ $\mathbb{C}^{N \times M \times N_{\mathrm{sc}} \times N_{\mathrm{sc}}}$ that represents the time-domain impulse responses. The $2 \mathrm{D}$ sections of $\mathcal{H}^{\text {Time }}$ are circularly symmetric matrices of the form [66]

$$
\mathcal{H}_{n m::}^{\text {Time }}=\operatorname{circulant}[\underbrace{\mathcal{H}_{n m:}^{\mathrm{BF}}}_{L \text { elements }}, \quad \underbrace{0, \cdots, 0}_{N_{\mathrm{sc}}-L \text { elements }}] \in \mathbb{C}^{N_{\mathrm{sc}} \times N_{\mathrm{sc}}} .
$$

Here, function circulant [.] generates a circulant matrix from an $N_{\mathrm{sc}}$-length vector.

Then, we represent the channel coefficients in the frequency-domain as a $3 \mathrm{D}$ tensor $\mathcal{H}^{\text {Freq }} \in \mathbb{C}^{N \times M \times N_{\mathrm{sc}}}$ as follows:

$$
\mathcal{H}_{n m:}^{\text {Freq }}=\operatorname{diag}\left[\mathbf{W}_{N_{\mathrm{sc}}} \mathcal{H}_{n m::}^{\text {Time }} \mathbf{W}_{N_{\mathrm{sc}}}^{\mathrm{H}}\right] \in \mathbb{C}^{N_{\mathrm{sc}}},
$$

where $\mathbf{W}_{N_{\mathrm{sc}}} \in \mathbb{C}^{N_{\mathrm{sc}} \times N_{\mathrm{sc}}}$ denotes the $N_{\mathrm{sc}} \times N_{\mathrm{sc}}$ discrete Fourier transform (DFT) matrix, and $\operatorname{diag}[\cdot]$ represents the diagonal elements of $\mathbf{W}_{N_{\mathrm{sc}}} \mathcal{H}_{n m::}^{\mathrm{Time}} \mathbf{W}_{N_{\mathrm{sc}}}^{\mathrm{H}} \cdot{ }^{3}$ Finally, the received symbol block at the transmission index $i$ and the subcarrier index $j\left(j=1, \cdots, N_{\mathrm{sc}}\right)$ is given by

$$
\mathbf{Y}(i, j)=\underbrace{\mathbf{H}(i, j)}_{(N \times M)} \underbrace{\mathbf{S}(i, j)}_{(M \times T)}+\underbrace{\mathbf{V}(i, j)}_{(N \times T)} \in \mathbb{C}^{N \times T},
$$

where we have $\mathbf{H}(i, j)=\mathcal{H}_{:: j}^{\text {Freq }}$. Fig. 1 shows the relation between $\mathcal{H}^{\text {Freq }}, \mathcal{H}_{n m:}^{\text {Freq }}$ and $\mathcal{H}_{:: j}^{\text {Freq }}$, where we have $N=$

${ }^{3}$ Note that the circulant matrix can be diagonalized by DFT matrices [66]. 
$M=4$. As shown in Fig. 1, $\mathcal{H}_{n m:}^{\text {Freq }}$ extracts the $N_{\mathrm{sc}}$-length vector associated with a specific index pair of $(n, m)$. The channel matrix $\mathbf{H}(i, j)=\mathcal{H}_{:: j}^{\text {Freq }}$ is generated from the $2 \mathrm{D}$ section of $\mathcal{H}^{\text {Freq }}$ associated with subcarrier index $j$. In Eq. (5), $\mathbf{S}(i, j) \in \mathbb{C}^{M \times T}$ represents the space-time codeword, and $\mathbf{V}(i, j) \in \mathbb{C}^{N \times T}$ denotes the additive white Gaussian noise (AWGN) that obeys a zero-mean complex-valued Gaussian distribution of $\mathcal{C N}\left(0, \sigma_{v}^{2}\right)$. According to Eq. (5), the SNR is defined by $\gamma=\mathrm{E}_{i, j}\left[\|\mathbf{S}(i, j)\|_{\mathrm{F}}^{2}\right] /\left(T \cdot \sigma_{v}^{2}\right)=1 / \sigma_{v}^{2}$.

\section{B. Time-Varying Channel Coefficients}

Based on the existing mmWave model of [63], we represent the time-varying channel coefficient $\lambda(i, l) \in \mathbb{C}$ in Eq. (1) as follows:

$$
\lambda(i, l)=\left\{\begin{array}{ll}
\mathcal{C N}(0,1) & (i=1) \\
r \lambda(i-1, l)+\sqrt{1-r^{2}} \mathcal{C N}(0,1) & (i>1)
\end{array},\right.
$$

which is modeled by a first-order AR process [67]. Here, the time-domain (TD) correlation function is defined by

$$
\rho(\tau)=\frac{\mathrm{E}_{i}\left[\lambda^{*}(i, l) \lambda(i+\tau, l)\right]}{\mathrm{E}_{i}\left[\lambda^{*}(i, l) \lambda(i, l)\right]}=r^{\tau},
$$

where $\tau \in \mathbb{Z}$ is a discrete time-lag. The process of determining an appropriate $r$ of Eq. (6) is described as follows. First, the coherence time $T_{c}$ is defined by the duration, where the TD correlation function is above 0.5 as follows: [68]

$$
T_{c}=0.423 \cdot \frac{v_{c}}{v} \cdot \frac{1}{f_{c}}
$$

Here, $v_{c}$ is the speed of light, $v>0$ is the speed of receiver ${ }^{4}$, and $f_{c}$ is the carrier frequency. In this paper, we assume that the carrier frequency is $f_{c}=60[\mathrm{GHz}]$, the DFT size is 512 , the OFDM sample rate is $2640[\mathrm{MHz}]$, and the normalized guard interval is $1 / 4$, which obey the IEEE 802.11 ad specifications [58]. Thus, the OFDM symbol duration is calculated by $T_{s}=512 /\left(2640 \cdot 10^{6}\right) \cdot(4+1) / 4[\mathrm{~s}]=242.4[\mathrm{~ns}]$, which includes the guard interval. Secondly, the number of OFDM symbols generated within the coherence time $T_{c}$ is then calculated by $N_{\mathrm{OFDM}}=\left\lfloor T_{c} / T_{s}\right\rfloor$. Since the TD correlation function $\rho(\tau)=r^{\tau}$ of Eq. (7) has to be above 0.5 between the time indices $i=1$ and $i=N_{\mathrm{OFDM}}$, we arrive at the following new constraint

$$
\begin{aligned}
r^{N_{\text {OFDM }}}=0.5 \Leftrightarrow r & =\exp \left(\frac{\log _{e} 0.5}{N_{\text {OFDM }}}\right) \\
& =\exp \left(-\frac{0.6931472}{\left\lfloor T_{c} /\left(242.4 \cdot 10^{-9}\right)\right\rfloor}\right) .
\end{aligned}
$$

According to Eq. (9), we find that the AR coefficient $r$ depends on the coherence time $T_{c}$, while directly depends on the mobile speed $v$. These relationships are exemplified in Table I.

\footnotetext{
${ }^{4}$ When $v=0$, we use $r=1$. This setup is equivalent to a quasi-static channel, where the channel coefficients are fixed within a frame length $W$.
}

TABLE I

TIME-VARYING CHANNEL PARAMETERS BASED ON THE IEEE 802.11 AD SPECIFICATIONS.

\begin{tabular}{|l||l|l|}
\hline Speed $v[\mathrm{~km} / \mathrm{h}]$ & Coherence time $T_{c}[\mathrm{~ms}]$ & AR coefficient $r$ \\
\hline \hline 1 & 7.608732584039999 & 0.999977915646254 \\
5 & 1.521746516808000 & 0.999889579590400 \\
10 & 0.760873258404000 & 0.999779136191148 \\
100 & 0.076087325840400 & 0.997787922524068 \\
\hline
\end{tabular}

\section{Conventional Square And Nonsquare DSTC}

The conventional square-matrix-based DSTC scheme of [38-41] generates a unitary matrix, multiplies it by the previous unitary matrix, and then transmits the multiplied matrix. Since the receiver knows that the received matrix is generated based on the previous matrix, it can detect the transmitted matrix without channel coefficients, where the previous matrix acts as if it was a pilot symbol block. By contrast, the conventional nonsquare-matrix-based DSTC scheme maps the unitary matrix onto a nonsquare matrix [52], and estimates the original unitary matrix from its mapped counterpart. Later in this section we will omit the subcarrier index $j$ for simplicity of notation.

\section{A. Square and Nonsquare Differential Encoding}

The $i$ th unitary matrix $\tilde{\mathbf{S}}(i) \in \mathbb{C}^{M \times M}$ is generated by differential encoding [38] as follows:

$$
\tilde{\mathbf{S}}(i)= \begin{cases}\mathbf{I}_{M} & (i \leq M / T) \\ \tilde{\mathbf{S}}(i-1) \mathbf{X}(i) & (i>M / T)\end{cases}
$$

where a data matrix $\mathbf{X}(i) \in \mathbb{C}^{M \times M}$ is associated with the $B$-length input bit sequence and satisfies $\mathbf{X}(i) \mathbf{X}(i)^{\mathrm{H}}=\mathbf{I}_{M} \cdot{ }^{5}$ Then, the $i$ th space-time matrix of Eq. (5) is generated by $\mathbf{S}(i)=\tilde{\mathbf{S}}(i) \mathbf{E}_{1} \in \mathbb{C}^{M \times T}$, where a basis $\mathbf{E}_{1} \in \mathbb{C}^{M \times T}$ converts an $M \times M$ matrix into an $M \times T$ matrix. Here, the basis set $\left\{\mathbf{E}_{1}, \cdots, \mathbf{E}_{M / T}\right\}$ is calculated by the DFT matrix as follows: [52]

$$
\tilde{\mathbf{I}}=\left[\mathbf{E}_{1}, \cdots, \mathbf{E}_{M / T}\right]=\operatorname{bdiag} \underbrace{\left[\mathbf{W}_{N_{b}}, \cdots, \mathbf{W}_{N_{b}}\right]}_{M / N_{b} \text { elements }} \in \mathbb{C}^{M \times M},
$$

where $N_{b}$ denotes the number of nonzero elements in each basis column. For the $M=T$ and $N_{b}=1$ scenario, $\tilde{\mathbf{I}}=\mathbf{E}_{1}$ is equal to $\mathbf{I}_{M}$, which is identical with the classic differential encoding. In Eq. (11), the DFT matrix is defined by

$\mathbf{W}_{M}=\frac{1}{\sqrt{M}}\left[\begin{array}{ccccc}1 & 1 & 1 & \cdots & 1 \\ 1 & \omega & \omega^{2} & \cdots & \omega^{M-1} \\ 1 & \omega^{2} & \omega^{4} & \cdots & \omega^{2(M-1)} \\ \vdots & \vdots & \vdots & \ddots & \vdots \\ 1 & \omega^{M-1} & \omega^{2(M-1)} & \cdots & \omega^{(M-1)(M-1)}\end{array}\right]$

\footnotetext{
${ }^{5}$ Based on the previous studies $[45,48,69]$, this differential encoding can be extended to support a scaled semi-unitary matrix, i.e., $\mathbf{X}(i) \mathbf{X}(i)^{\mathrm{H}}=$ $\beta \mathbf{I}_{M}$, where $\beta$ is a scalar. In our simulations, we have observed that this extension causes severe error propagations due to the recursive construction of Eq. (13). As will be shown in Section IV-A, the resultant constellation following $\mathbf{X}(i) \mathbf{X}(i)^{\mathrm{H}}=\mathbf{I}_{M}$ is similar to the star-QAM signaling.
} 
where we have $\omega=\exp (-2 \pi \jmath / M)$, and $\jmath$ denotes the imaginary number, i.e., $j^{2}=-1$.

As a reference symbol, during $1 \leq i \leq M / T$, $\tilde{\mathbf{I}}$ is transmitted over $M$ symbol duration, both for the square scenario of $M=T$ and nonsquare case of $M>T$. Then, for the $M / T+1 \leq i \leq W / T$ transmission indices, $W / T-M / T$ data symbols $\mathbf{S}(i) \in \mathbb{C}^{M \times T}$ are transmitted, where $W$ denotes the frame length. The ideal transmission rate is calculated by $R=B / T$, and the effective transmission rate is $R^{\mathrm{eff}}=(1-M / W) \cdot R$. The conventional nonsquare scheme of [51] transmits reference symbols $\tilde{\mathbf{I}}$ every $100 M$ blocks, i.e., we have $W=100 M$. In our simulations, the channel coefficients are randomized every $W=1000 M$ blocks. Thus, the rate loss is kept below $0.1 \%$, and the periodic reference symbol insertions are nearly negligible.

Let us consider for example the family of nonsquare Alamouti codes $[38,70]$ having $(M, T, R)=(2,1,2)$. At the $i=M / T+1$ transmission index, a BPSK-aided square spacetime codeword $\tilde{\mathbf{S}}(i)=\tilde{\mathbf{S}}(i-1) \mathbf{X}(i)=\mathbf{I}_{M} \mathbf{X}(i)=\mathbf{X}(i)$ is given by [70]

$$
\tilde{\mathbf{S}}(i)=\frac{1}{\sqrt{2}}\left[\begin{array}{cc}
1 & -1 \\
1 & 1
\end{array}\right]
$$

Then, the DFT basis set having $M=2$ and $N_{b}=2$ is given by

$$
\left\{\mathbf{E}_{1}, \mathbf{E}_{2}\right\}=\left\{\frac{1}{\sqrt{2}}\left[\begin{array}{l}
1 \\
1
\end{array}\right], \frac{1}{\sqrt{2}}\left[\begin{array}{c}
1 \\
-1
\end{array}\right]\right\},
$$

which corresponds to the first and second columns of the DFT matrix. Finally, a nonsquare codeword is calculated by

$$
\mathbf{S}(i)=\tilde{\mathbf{S}}(i) \mathbf{E}_{1}=\frac{1}{\sqrt{2}}\left[\begin{array}{cc}
1 & -1 \\
1 & 1
\end{array}\right] \cdot \frac{1}{\sqrt{2}}\left[\begin{array}{l}
1 \\
1
\end{array}\right]=\left[\begin{array}{l}
0 \\
1
\end{array}\right] .
$$

Further examples are found in [52].

\section{B. Noncoherent Detection}

The $i$ th data matrix $\mathbf{X}(i)$ is estimated by minimizing the following criterion [52]

$$
\hat{\mathbf{X}}(i)=\arg \min _{\mathbf{X}}\left\|\mathbf{Y}(i)-\hat{\mathbf{Y}}(i-1) \mathbf{X} \mathbf{E}_{1}\right\|_{\mathbf{F}}^{2},
$$

which is known as the maximum likelihood (ML) detector. Here, we have the difference equation of

$$
\hat{\mathbf{Y}}(i)=\left\{\begin{array}{ll}
\sum_{k=1}^{M / T} \mathbf{Y}(k) \mathbf{E}_{k}^{\mathrm{H}} & (i=M / T) \\
\mathbf{Y}(i) \mathbf{E}^{(1-\alpha)}+\hat{\mathbf{Y}}(i-1) \hat{\mathbf{X}}(i) \mathbf{E}^{(\alpha)} & (i>M / T)
\end{array} .\right.
$$

Ideally, $\hat{\mathbf{Y}}(i)$ approaches $\mathbf{H}(i) \tilde{\mathbf{S}}(i) \in \mathbb{C}^{M \times M}$, which is estimated from the mapped symbols $\mathbf{H}(i) \tilde{\mathbf{S}}(i) \mathbf{E}_{1} \in \mathbb{C}^{M \times T}$. In Eq. (13), the constant matrices are defined by $\mathbf{E}^{(1-\alpha)}=$ $(1-\alpha) \mathbf{E}_{1}^{\mathrm{H}}$ and $\mathbf{E}^{(\alpha)}=\mathbf{I}_{M}-\mathbf{E}_{1} \mathbf{E}^{(1-\alpha)}$. Here, $\alpha$ is a forgetting factor that determines a combination ratio between the newly received symbol $\mathbf{Y}(i)$ and the previous symbols $\mathbf{Y}(i-1), \cdots, \mathbf{Y}(1)$. This single factor $\alpha$ is designed for minimizing a specific squared error [51,52], which will be detailed in Section III-C. Note that for the $M=T$ and $\alpha=0$ scenario, Eq. (12) becomes equivalent to the conventional square-matrix-based detector, i.e.,

$$
\hat{\mathbf{X}}(i)=\arg \min _{\mathbf{X}}\|\mathbf{Y}(i)-\mathbf{Y}(i-1) \mathbf{X}\|_{\mathbf{F}}^{2} .
$$

When relying on a complex-valued basis set, the ML detection complexity of Eq. (12) per receive subarray is given by [52]

$$
2^{R T+1}\left[2 \cdot \max \left(N_{b}, N_{c}\right)+1\right]+\frac{4 M}{T}\left(N_{b}+N_{c}\right)+4 N_{b},
$$

where $N_{c}$ denotes the number of nonzero elements in each column of $\tilde{\mathbf{S}}(i)$. Then, the number of activated subarrays is calculated by $P=\max \left(N_{b}, N_{c}\right)$ [52], which is equal to the number of nonzero elements in each column of $\tilde{\mathbf{S}}(i) \mathbf{E}_{1}$. Eq. (14) is lower-bounded by $\Omega\left(2^{R T} \cdot \max \left(N_{b}, N_{c}\right)\right)=\Omega\left(2^{R T} \cdot P\right){ }^{6}$

\section{Forgetting Factor Design}

The conventional detector of Eq. (12) requires a carefullydesigned $\alpha \in \mathbb{R}(0<\alpha<1)$, which determines the overall system performance. In the previous studies [51,52], the forgetting factor $\alpha$ is designed for minimizing the following squared error:

$$
\alpha_{c}=\arg \min _{\alpha \in(0,1)} J_{\mathrm{MSE}}
$$

where $J_{\mathrm{MSE}}$ is defined as [51]

$$
J_{\mathrm{MSE}}=\frac{T}{W-M} \frac{1}{N M} \sum_{i=M / T+1}^{W / T} \mathrm{E}\left[\|\hat{\mathbf{Y}}(i)-\mathbf{H}(i) \tilde{\mathbf{S}}(i)\|_{\mathrm{F}}^{2}\right] .
$$

Later, we will represent the forgetting factor designed for quasi-static channels by $\alpha_{c}$. Here, it is a time-consuming task to obtain $\alpha_{c}$, because Eq. (16) should be averaged over random $\mathbf{H}(i), \mathbf{V}(i)$, and $\mathbf{X}(i)$ matrices, and the forgetting factor $\alpha_{c}$ has to be searched on a finely grained discrete set such as $\alpha=0.01,0.02, \cdots, 0.99$. In case we assume quasi-static Rayleigh fading channels, Eq. (15) can be simplified, and the forgetting factor $\alpha_{c}$ can be obtained by solving [51]

$$
\begin{array}{r}
2(K-1) \alpha_{c}^{2 K+2}+\alpha_{c}^{2 K+1}-(2 K+1) \alpha_{c}^{2 K} \\
-K \alpha_{c}^{3}+(K+2) \alpha_{c}^{2}+(K-1) \alpha_{c}-K+1=0,
\end{array}
$$

where we have $K=W / M$. As seen in Eq. (17), the forgetting factor $\alpha_{c}$ is dependent both on the number of transmit subarrays $M$ and on the frame length $W$. Since this simple criterion cannot be applied to time-varying channels, we have to rely on $J_{\mathrm{MSE}}$ of Eq. (16), which will be addressed in Section IV.

\section{Proposed Adaptive Differential GSM}

The conventional nonsquare-matrix-based scheme of [52] relies on the DFT basis and on the static forgetting factor, as introduced in Sections III-A and III-C, which result in performance loss, especially for time-varying mmWave channels. Thus, in this section we propose a novel basis set and a novel forgetting factor design. Later, the transmission and subcarrier indices $i$ and $j$ are omitted, if they are not necessary.

${ }^{6} \Omega(\cdot)$ denotes the Donald Knuth's big Omega notation [71]. 


\section{A. Gram-Schmidt Process Aided Basis Design}

The DFT basis imposes the additional constraint of $\left\lfloor M / N_{b}\right\rfloor=M / N_{b}$. For example, if we consider the $M=16$ case, $N_{b}$ is limited to $1,2,4,8$ and 16 . Furthermore, the DFT basis is unable to support some DSTC schemes, such as the algebraic DSM (ADSM) [48] having $O>1$ independent PSK symbols. This problem is encountered when the DFT projection is a non-injective function. Specifically, let us consider the $(M, T)=(4,1)$ and $N_{b}=2$ case. Here, the DFT basis is calculated by $\mathbf{E}_{1}=\left[\begin{array}{llll}1 & 1 & 0 & 0\end{array}\right]^{\mathrm{T}} / \sqrt{2}$. Through the projection of $\tilde{\mathbf{S}}$ to $\tilde{\mathbf{S}} \mathbf{E}_{1}$, only the first and second columns of $\tilde{\mathbf{S}}$ are extracted, while the third and fourth columns are discarded. Since the discarded columns include data-carrying symbols, this setup results in severe performance loss. Therefore, multiplying the basis $\mathbf{E}_{1}$ has to be an injective mapping.

We propose a basis set relying on the Gram-Schmidt process (GSP) [56], which imposes no limitation on the number of nonzero elements $N_{b}$. As seen in Eq. (14), the ML detection complexity can be improved by reducing $N_{b}$ and $N_{c}$. In mmWave communications, the number of activated subarrays $P=\max \left(N_{b}, N_{c}\right)$ determines the $\mathrm{BF}$ gain. Here, we have a trade-off between the ML complexity and the BF gain, which can be controlled by using a flexible GSP-aided basis.

When assuming perfect CSI at the receiver, the pairwiseerror probability (PEP) between the two arbitrary symbols $\tilde{\mathbf{S}}^{(f)} \mathbf{E}_{1}$ and $\tilde{\mathbf{S}}^{(g)} \mathbf{E}_{1}$ for $1 \leq f<g \leq 2^{B}$ is upper bounded by $[56,72]$

$$
\operatorname{PEP}(f \rightarrow g) \leq \frac{1}{d(f, g)^{N}}\left(\frac{1}{4 \sigma_{v}^{2}}\right)^{-m^{\prime} N},
$$

where $m^{\prime}$ represents the minimum rank of the Hermitian matrix $\mathbf{C}(f, g)=\left(\tilde{\mathbf{S}}^{(f)}-\tilde{\mathbf{S}}^{(g)}\right) \mathbf{E}_{1} \mathbf{E}_{1}^{\mathrm{H}}\left(\tilde{\mathbf{S}}^{(f)}-\tilde{\mathbf{S}}^{(g)}\right)^{\mathrm{H}} \in$ $\mathbb{C}^{M \times M}$. In Eq. (18), the diversity order is given by $D=m^{\prime} N$ and the coding gain is given by the Euclidean distance of $d(f, g)=\prod_{m=1}^{m^{\prime}} \mu_{m} \in \mathbb{R}$, where $\mu_{m}$ represents the $m$ th eigenvalue of $\mathbf{C}(f, g)$.

Although Eq. (18) is derived for coherent detection scenarios, this upper bound is also valid for designing differential codewords $[44,73]$. Thus, we design the basis $\mathbf{E}_{1} \in \mathbb{C}^{M \times T}$ so as to maximize the minimum Euclidean distance (MED) of

$$
\mathrm{MED}=\min _{1 \leq f<g \leq 2^{B}} d(f, g) .
$$

Here, $\mathbf{E}_{1}$ has $N_{b}$ nonzero elements in each column, and satisfies the constraint of $\mathbf{E}_{1}^{\mathrm{H}} \mathbf{E}_{1}=\mathbf{I}_{T}$, which implies $\left\|\mathbf{E}_{1}\right\|_{\mathrm{F}}^{2}=$ trace $\left(\mathbf{E}_{1}^{\mathrm{H}} \mathbf{E}_{1}\right)=T$. Some of the GSP basis sets designed are exemplified in Appendix A.

After designing the first basis $\mathbf{E}_{1}$, the other bases $\mathbf{E}_{2}, \cdots, \mathbf{E}_{M / T}$ are generated by the GSP, where the dense DFT bases of Section III-A are exploited. The conventional DFT basis set having $N_{b}=M$ will be denoted by $\left\{\mathbf{E}_{1}^{\prime}, \cdots, \mathbf{E}_{M / T}^{\prime}\right\}$ in this section. Based on the first basis $\mathbf{E}_{1}$ designed, the next basis $\mathbf{E}_{k}$, whose index is increased from $k=2$ to $M / T$, is expressed by

$$
\mathbf{E}_{k}=\left(\mathbf{I}_{M}-\sum_{k^{\prime}=1}^{k-1} \mathbf{E}_{k^{\prime}} \mathbf{E}_{k^{\prime}}^{\mathrm{H}}\right) \mathbf{E}_{k}^{\prime}
$$

and then normalized by $\mathbf{E}_{k}:=\sqrt{T} \cdot \mathbf{E}_{k} /\left\|\mathbf{E}_{k}\right\|_{\mathrm{F}}$. This normalization is required to satisfy the norm constraint of $\left\|\mathbf{E}_{k}\right\|_{\mathrm{F}}^{2}=T$. For example, the GSP basis set for the ADSM scheme having $(M, T, O, L)=(4,1,2,16)$ and $N_{b}=2$ is given by

$$
\begin{aligned}
\tilde{\mathbf{I}} & =\left[\mathbf{E}_{1}, \mathbf{E}_{2}, \mathbf{E}_{3}, \mathbf{E}_{4}\right] \\
& =\left[\begin{array}{cccc}
0.00 & 0.56 e^{\jmath 0.00 \pi} & 0.44 e^{\jmath 1.98 \pi} & 0.70 e^{\jmath 1.98 \pi} \\
0.76 e^{\jmath 0.95 \pi} & 0.40 e^{\jmath 1.26 \pi} & 0.23 e^{\jmath 1.31 \pi} & 0.46 e^{\jmath 0.26 \pi} \\
0.00 & 0.56 e^{\jmath 1.00 \pi} & 0.82 e^{\jmath 0.01 \pi} & 0.09 e^{\jmath 1.20 \pi} \\
0.65 e^{\jmath 1.39 \pi} & 0.47 e^{\jmath 0.70 \pi} & 0.27 e^{\jmath 0.75 \pi} & 0.54 e^{\jmath 1.70 \pi}
\end{array}\right],
\end{aligned}
$$

which is a unitary matrix. A part of the nonsquare ADSM codewords $\tilde{\mathbf{S}} \mathbf{E}_{1}$ result in

$$
\left[\begin{array}{c}
0.65 e^{\jmath 1.64 \pi} \\
0.00 \\
0.76 e^{\jmath 1.08 \pi} \\
0.00
\end{array}\right],\left[\begin{array}{c}
0.00 \\
0.76 e^{\jmath 0.95 \pi} \\
0.00 \\
0.65 e^{\jmath 1.39 \pi}
\end{array}\right],\left[\begin{array}{c}
0.76 e^{\jmath 1.08 \pi} \\
0.00 \\
0.00 \\
0.65 e^{\jmath 1.39 \pi}
\end{array}\right],\left[\begin{array}{c}
0.00 \\
0.76 e^{j 0.95 \pi} \\
0.65 e^{\jmath 1.52 \pi} \\
0.00
\end{array}\right],
$$

all of which contain $P=\max \left(N_{b}, N_{c}\right)=\max (2,1)=2$ nonzero elements in each column. This setup is equivalent to the differential counterpart of the conventional GSM [31]. It is worth noting that all the TAs are selected with an equal probability due to the permutation matrix of the ADSM scheme, without relying on the sophisticated algorithm proposed in [74].

Furthermore, Fig. 2 shows illustrative examples for the nonsquare ADSM symbols having $(M, T, O, L)=(16,1,2,4)$, where three growing MEDs were considered for characterizing the MED-maximization design process. Here, we used the GSP basis having $N_{b}=12$ and generated $1000 \cdot M$ number of random codewords. The best GSP basis achieving $\mathrm{MED}=0.877168$ is given in Appendix A. Since a fraction of $1-N_{b} / M=1-12 / 16=25 \%$ of the symbols are zero, we omitted them for clear illustration. As shown in Fig. 2, upon growing the MED, the nonsquare ADSM symbols converged on a spiral pattern, and the cardinality of these symbols remained finite for all the cases under the multiplication-based differential encoding. Most of the conventional differential MIMO schemes generate infinite and near-continuous constellation, which imposes additional complexity on the transmitter $[45,46]$. Our GSP basis is capable of avoiding this infinitecardinality problem.

\section{B. Adaptive Forgetting Factor Design}

Since the evaluation of the conventional design criterion in Eq. (15) is a high-complexity time-consuming task for the time-varying scenarios, we propose a low-complexity alternative. First, the $J_{\mathrm{MSE}}$ definition of Eq. (16) is transformed into

$$
J_{\mathrm{MSE}}=\frac{T}{W-M} \frac{1}{N T} \sum_{i=M / T+1}^{W / T} \mathrm{E}\left[\left\|\hat{\mathbf{Y}}(i) \mathbf{E}_{1}-\mathbf{H}(i) \tilde{\mathbf{S}}(i) \mathbf{E}_{1}\right\|_{\mathrm{F}}^{2}\right]
$$




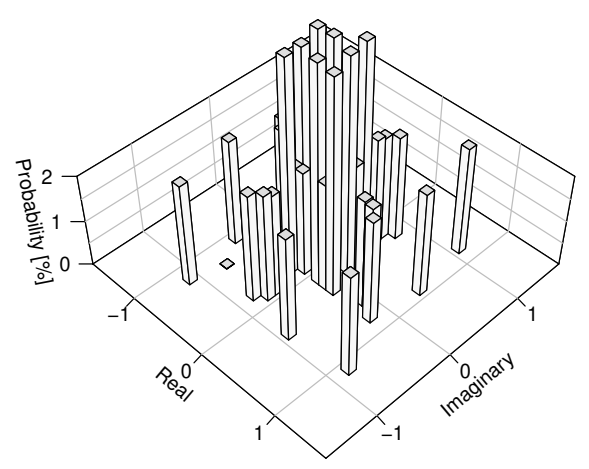

(a) $\mathrm{MED}=0.184695$.

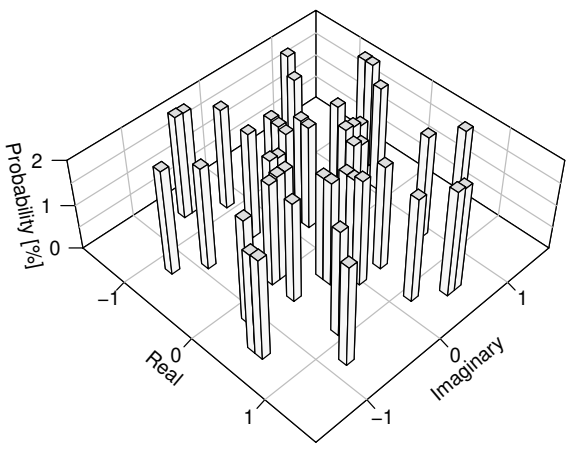

(b) $\mathrm{MED}=0.363259$.

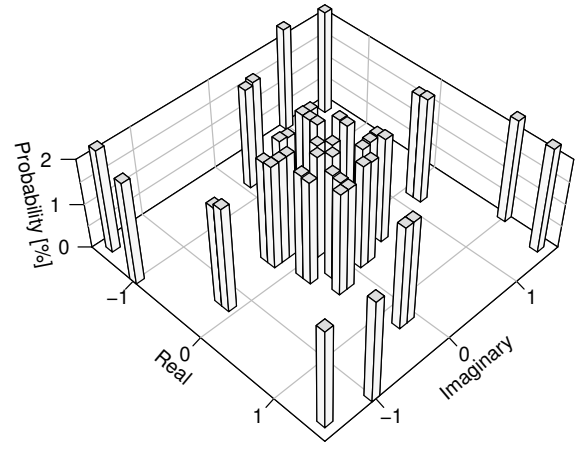

(c) $\mathrm{MED}=0.877168$ (best).

Fig. 2. The histogram of nonsquare ADSM constellation for the $(M, T, O, L)=(16,1,2,4)$ case, where the GSP basis having $N_{b}=12$ was considered and $1000 \cdot M$ number of random codewords were generated.

because the average of the Frobenius norm in Eq. (16) is equivalent to ${ }^{7}$

$$
\begin{aligned}
& \mathrm{E}\left[\|\hat{\mathbf{Y}}(i)-\mathbf{H}(i) \tilde{\mathbf{S}}(i)\|_{\mathrm{F}}^{2}\right]=\mathrm{E}\left[\|(\hat{\mathbf{Y}}(i)-\mathbf{H}(i) \tilde{\mathbf{S}}(i)) \tilde{\mathbf{I}}\|_{\mathrm{F}}^{2}\right] \\
= & \frac{M}{T} \cdot \mathrm{E}\left[\left\|\hat{\mathbf{Y}}(i) \mathbf{E}_{1}-\mathbf{H}(i) \tilde{\mathbf{S}}(i) \mathbf{E}_{1}\right\|_{\mathrm{F}}^{2}\right] .
\end{aligned}
$$

Here, we have the relationships of $\mathbf{Y}(i)=\mathbf{H}(i) \tilde{\mathbf{S}}(i) \mathbf{E}_{1}+$ $\mathbf{V}(i)$ and $\hat{\mathbf{Y}}(i)=(1-\alpha) \mathbf{Y}(i) \mathbf{E}_{1}^{\mathrm{H}}+\hat{\mathbf{Y}}(i-1) \hat{\mathbf{X}}(i)\left(\mathbf{I}_{M}-(1-\right.$ $\left.\alpha) \mathbf{E}_{1} \mathbf{E}_{1}^{\mathrm{H}}\right)$. Thus, the Frobenius norm of Eq. (21) can be further simplified to

$$
\begin{aligned}
& \hat{\mathbf{Y}}(i) \mathbf{E}_{1}-\mathbf{H}(i) \tilde{\mathbf{S}}(i) \mathbf{E}_{1} \\
= & \hat{\mathbf{Y}}(i) \mathbf{E}_{1}-\mathbf{Y}(i)+\mathbf{V}(i) \\
= & -\alpha \underbrace{\left(\mathbf{Y}(i)-\hat{\mathbf{Y}}(i-1) \hat{\mathbf{X}}(i) \mathbf{E}_{1}\right)}_{\equiv \mathbf{D}(i)}+\mathbf{V}(i) .
\end{aligned}
$$

Later, we represent $\mathbf{Y}(i)-\hat{\mathbf{Y}}(i-1) \hat{\mathbf{X}}(i) \mathbf{E}_{1}$ as $\mathbf{D}(i)$ for simplicity. Then, $J_{\mathrm{MSE}} \equiv f(\alpha)$ can be transformed into

$$
\begin{array}{r}
f(\alpha)=\frac{T}{W-M} \frac{1}{N T} \mathrm{E}\left[\alpha^{2} \sum_{i=M / T+1}^{W / T}\|\mathbf{D}(i)\|_{\mathrm{F}}^{2}\right. \\
\left.-2 \alpha \sum_{i=M / T+1}^{W / T} \operatorname{Re}\left[\operatorname{tr}\left(\mathbf{V}(i)^{\mathrm{H}} \mathbf{D}(i)\right)\right]+\sum_{i=M / T+1}^{W / T}\|\mathbf{V}(i)\|_{\mathrm{F}}^{2}\right] .
\end{array}
$$

The derivatives of $f(\alpha)$ with respect to $\alpha$ are calculated by

$$
\begin{array}{r}
f^{\prime}(\alpha)=\frac{T}{W-M} \frac{1}{N T} \mathrm{E}\left[2 \alpha \sum_{i=M / T+1}^{W / T}\|\mathbf{D}(i)\|_{\mathrm{F}}^{2}\right. \\
-2 \sum_{i=M / T+1}^{W / T} \operatorname{Re}\left[\operatorname{tr}\left(\mathbf{V}(i)^{\mathrm{H}} \mathbf{D}(i)\right)\right]
\end{array}
$$

and

$$
f^{\prime \prime}(\alpha)=\frac{T}{W-M} \frac{1}{N T} \mathrm{E}\left[2 \sum_{i=M / T+1}^{W / T}\|\mathbf{D}(i)\|_{\mathrm{F}}^{2}\right] .
$$

\footnotetext{
${ }^{7}$ This transformation is valid when the average of the error matrix is zero, i.e., $\mathrm{E}[\hat{\mathbf{Y}}(i)-\mathbf{H}(i) \tilde{\mathbf{S}}(i)]=\mathbf{0}$. The error matrix $\hat{\mathbf{Y}}(i)-\mathbf{H}(i) \tilde{\mathbf{S}}(i)$ is known to follow the Gaussian distribution [52].
}

Since we have $f^{\prime \prime}(\alpha) \geq 0$, solving $f^{\prime}(\alpha)=0$ yields the best forgetting factor in terms of Eq. (21) as follows:

$$
\alpha=\mathrm{E}\left[\frac{\sum_{i=M / T+1}^{W / T} \operatorname{Re}\left[\operatorname{tr}\left(\mathbf{V}(i)^{\mathrm{H}} \mathbf{D}(i)\right)\right]}{\sum_{i=M / T+1}^{W / T}\|\mathbf{D}(i)\|_{\mathrm{F}}^{2}}\right] .
$$

However, the AWGN term $\mathbf{V}(i)$ appeared in Eq. (24) is not available at the receiver, as defined in Eq. (5). Then, we transform $\operatorname{Re}\left[\operatorname{tr}\left(\mathbf{V}(i)^{\mathrm{H}} \mathbf{D}(i)\right)\right]$ into

$$
\begin{gathered}
\operatorname{Re}\left[\operatorname{tr}\left(\mathbf{V}(i)^{\mathrm{H}} \mathbf{D}(i)\right)\right]=\operatorname{Re}\left[\operatorname{tr}\left(\mathbf{V}(i)^{\mathrm{H}} \mathbf{H}(i) \tilde{\mathbf{S}}(i) \mathbf{E}_{1}\right)\right] \\
-\operatorname{Re}\left[\operatorname{tr}\left(\mathbf{V}(i)^{\mathrm{H}} \hat{\mathbf{Y}}(i-1) \hat{\mathbf{X}}(i) \mathbf{E}_{1}\right)\right]+\operatorname{Re}\left[\operatorname{tr}\left(\mathbf{V}(i)^{\mathrm{H}} \mathbf{V}(i)\right)\right] .
\end{gathered}
$$

Furthermore, we use the assumption of $\hat{\mathbf{Y}}(i-1) \hat{\mathbf{X}}(i) \approx \mathbf{H}(i-$ 1) $\tilde{\mathbf{S}}(i-1) \hat{\mathbf{X}}(i) \approx \mathbf{H}(i-1) \tilde{\mathbf{S}}(i) \approx \mathbf{H}(i) \tilde{\mathbf{S}}(i)$ as follows:

$\operatorname{Re}\left[\operatorname{tr}\left(\mathbf{V}(i)^{\mathrm{H}} \mathbf{D}(i)\right)\right]$

$=\operatorname{Re}\left[\operatorname{tr}\left(\mathbf{V}(i)^{\mathrm{H}} \mathbf{H}(i) \tilde{\mathbf{S}}(i) \mathbf{E}_{1}\right)\right]-\operatorname{Re}\left[\operatorname{tr}\left(\mathbf{V}(i)^{\mathrm{H}} \mathbf{H}(i) \tilde{\mathbf{S}}(i) \mathbf{E}_{1}\right)\right]$

$+\operatorname{Re}\left[\operatorname{tr}\left(\mathbf{V}(i)^{\mathrm{H}} \mathbf{V}(i)\right)\right] \approx \operatorname{Re}\left[\operatorname{tr}\left(\mathbf{V}(i)^{\mathrm{H}} \mathbf{V}(i)\right)\right]$.

Here, the mean of Eq. (25) converges to $\mathrm{E}\left[\operatorname{Re}\left[\operatorname{tr}\left(\mathbf{V}(i)^{\mathrm{H}} \mathbf{V}(i)\right)\right]\right]=N \cdot T \cdot \sigma_{v}^{2}$. Thus, Eq. (24) is approximated by

$$
\alpha_{p}=\frac{\left(\frac{W}{T}-\frac{M}{T}\right) \cdot N \cdot T \cdot \sigma_{v}^{2}}{\mathrm{E}\left[\sum_{i=M / T+1}^{W / T}\|\mathbf{D}(i)\|_{\mathrm{F}}^{2}\right]} .
$$

Based on Eq. (26), we propose a pair of low-complexity adaptive schemes for the time-invariant and time-varying channels, respectively. Furthermore, we introduce an adaptive forgetting factor $\alpha(i)$, and $\hat{\mathbf{Y}}(i)$ of Eq. (13) for $i>M / T$ is redefined as follows:

$$
\hat{\mathbf{Y}}(i)=(1-\alpha(i)) \mathbf{D}(i) \mathbf{E}_{1}^{\mathrm{H}}+\hat{\mathbf{Y}}(i-1) \hat{\mathbf{X}}(i) .
$$

1) Time-Invariant Channels: In quasi-static channels, the adaptive forgetting factor $\alpha_{q}(i)$ is calculated from Eq. (26) as follows:

$$
\alpha_{q}(i)=\frac{\left(i-\frac{M}{T}\right) \cdot N \cdot T \cdot \sigma_{v}^{2}}{\sum_{i^{\prime}=M / T+1}^{i}\left\|\mathbf{D}\left(i^{\prime}\right)\right\|_{\mathrm{F}}^{2}} .
$$

Eq. (28) contains the received symbols spanning from the $(M / T+1)$-st block to the $i$-th block, which may overflow 


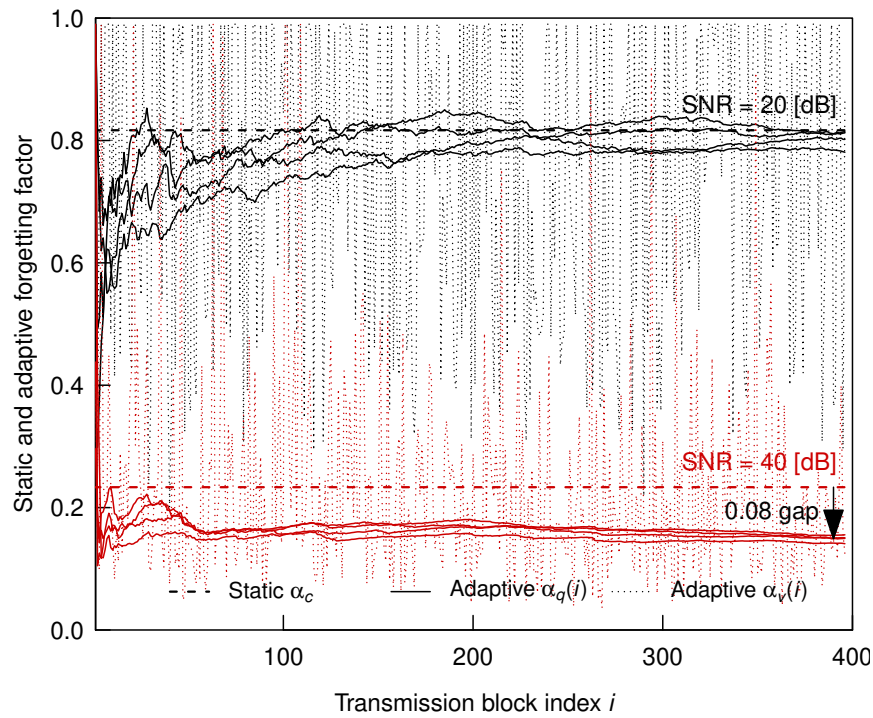

Fig. 3. Static and adaptive forgetting factor comparisons, where the numbers of transmit and receive subarrays were $M=N=4$, the transmission rate was $R=12[\mathrm{bps} / \mathrm{Hz}]$, and the mobile speed was $v=1[\mathrm{~km} / \mathrm{h}]$.

when the transmission index is high. Thus, Eq. (28) is rewritten by

$$
\frac{1}{\alpha_{q}(i)}=\left(1-\frac{1}{i-\frac{M}{T}}\right) \frac{1}{\alpha_{q}(i-1)}+\frac{1}{i-\frac{M}{T}} \cdot \frac{\|\mathbf{D}(i)\|_{\mathrm{F}}^{2}}{N \cdot T \cdot \sigma_{v}^{2}},
$$

which only relies on the current $\mathbf{D}(i)$ and on the previous forgetting factor $\alpha_{q}(i-1)$. This reduces the space complexity ${ }^{8}$ as compared to Eq. (26). When the transmission index $i$ is sufficiently high, $\frac{1}{i-M / T}$ approaches 0 , and $\alpha_{q}(i)$ converges to a finite value, i.e., $\alpha_{q}(i)=\alpha_{q}(i-1)$.

2) Time-Varying Channels: In time-varying channels, we only consider the recent two blocks rather than all the $i-$ $M / T$ blocks. Specifically, the adaptive forgetting factor $\alpha_{v}(i)$ is given by

$$
\alpha_{v}(i)=\frac{N \cdot T \cdot \sigma_{v}^{2}}{\|\mathbf{D}(i)\|_{\mathrm{F}}^{2}}
$$

Since we have $\mathbf{D}(i)=\mathbf{Y}(i)-\hat{\mathbf{Y}}(i-1) \hat{\mathbf{X}}(i) \mathbf{E}_{1}$, Eq. (30) only relies on the $i$-th and $(i-1)$-st received blocks, which is different from $\alpha_{q}(i)$ of Eq. (29).

The forgetting factor derived may violate the domain of definition $0<\alpha(i)<1$ due to the approximation assumed in Eq. (25). Thus, we use its clipped counterpart of $\min [\max [\alpha(i), 0.01], 0.99]$ in our simulations. Note that the forgetting factors $\alpha_{q}(i)$ and $\alpha_{v}(i)$ proposed in Eqs. (29) and (30) are also applicable to general channel models such as time-invariant and time-varying Rayleigh, Rician, and Gamma-Gamma fading.

Fig. 3 exemplifies the static and adaptive forgetting factors $\alpha_{c}, \alpha_{q}(i)$ and $\alpha_{v}(i)$, where the mobile speed was $v=1[\mathrm{~km} / \mathrm{h}]$ and the SNR was set to $\gamma=20$ and $40[\mathrm{~dB}]$. As shown in

\footnotetext{
${ }^{8}$ Here, the space complexity is defined by the size of work memory consumed by equations.
}

Fig. 3, the adaptive forgetting factor $\alpha_{v}(i)$ fluctuated against the block index $i$, while $\alpha_{q}(i)$ converged to a near-constant value. Ideally, $\alpha_{q}(i)$ has to converge to $\alpha_{c}$ that minimizes $J_{\mathrm{MSE}}$ of Eq. (16). For the SNR of $\gamma=20[\mathrm{~dB}]$ case, $\alpha_{q}(i)$ converged to $\alpha_{c}$, while we observed a gap of about 0.08 at $i=400$ for the SNR $\gamma=40[\mathrm{~dB}]$ case. This slight gap was imposed by the approximation of Eq. (25). It is worth noting that the proposed forgetting factors decreased as the SNR increased, while the conventional criterion of Eq. (17) only considered high SNRs and it was unable to support the SNR shift.

\section{PERformance Comparisons}

In this section, we compare the proposed scheme both to the conventional differential orthogonal space-time coding (DOSTC) $[38,70]$ and to the differential unitary coding (DUC) [41] as well as to the conventional coherent BLAST [9] and GSM [31,75] schemes that have perfect estimates of CSI (PCSI) in terms of their MED, bit error ratio (BER), and $J_{\mathrm{MSE}}$. Later, the abbreviation of the square-matrix-based schemes starts with "S-", while that of the nonsquare-matrix-based schemes starts with "N-". For the $M \cdot R \leq 32$ case, we did our best to simulate the conventional S-DSTC schemes, although its computation time is so large that it is difficult to reproduce the same results in a typical computing environment. ${ }^{9}$ Since achieving a high throughput is crucial for mmWave communications, we only focus on the high-rate scenarios, where the transmission rate is increased up to $R=16$ [bps/Hz].

As in the IEEE 802.11ad specifications [58], the number of data subcarriers was $N_{\mathrm{sc}}=339 .{ }^{10}$ The number of TA and RA elements was set to four times the number of subarrays, i.e., we have $M_{\mathrm{T}}=M_{\mathrm{R}}=4 M=4 N$, which were used for ABF. We considered both stationary and non-stationary scenarios, where the mobile speed was increased from $v=0$ to $v=100[\mathrm{~km} / \mathrm{h}]$. The number of multipath components was $L=M$, where the largest setup considered was $M=16 .{ }^{11}$ We basically used the GSP basis for the N-ADSM scheme, and the DFT basis for the N-DOSTC and the N-DUC schemes. The GSP bases used in our simulations are provided in Appendix A, and the DUC constellation factors are provided in Appendix B. Additionally, the number of nonzero elements in each codeword column is denoted by $P$.

\section{A. Small-Scale Scenarios}

First, we conducted MED and BER comparisons for smallscale scenarios, where the numbers of transmit and receive subarrays were smaller than $M=N \leq 4$.

Fig. 4 shows the MED comparisons between the conventional square schemes and the proposed nonsquare counterparts, where the basis $\mathbf{E}_{1}$ mapped both the DOSTC [38] and

\footnotetext{
${ }^{9}$ It is worth noting that for the $M \cdot R>32$ case the number of codewords exceeds $2^{32}$ and $2^{32}$-length array of $M \times M$ complex matrices may overflow.

${ }^{10}$ The BER performance unchanged upon increasing $N_{\text {sc }}$ because we assumed that the cyclic prefix length was larger than the maximum multipath delay.

${ }^{11}$ According to the survey of $[4$, Table X], some channel models assume that the number of multipath components obeys the Poisson distribution having the mean of $1.8,1.9,9,10$ and 18 .
} 


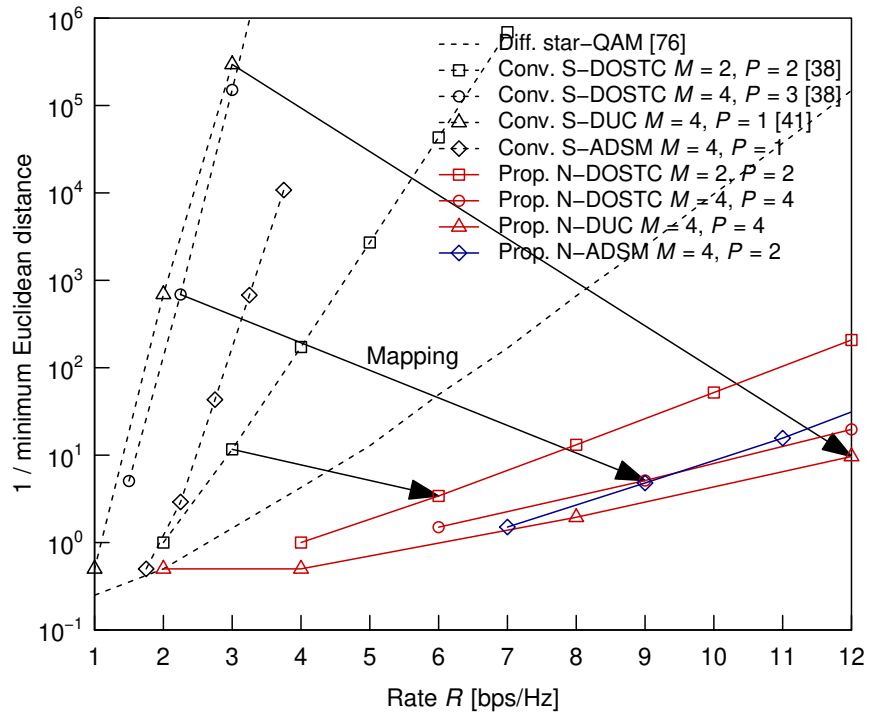

Fig. 4. MED of Eq. (19) comparisons of the proposed nonsquare and the conventional square schemes, where the number of transmit subarrays was $M=2$ and 4 .

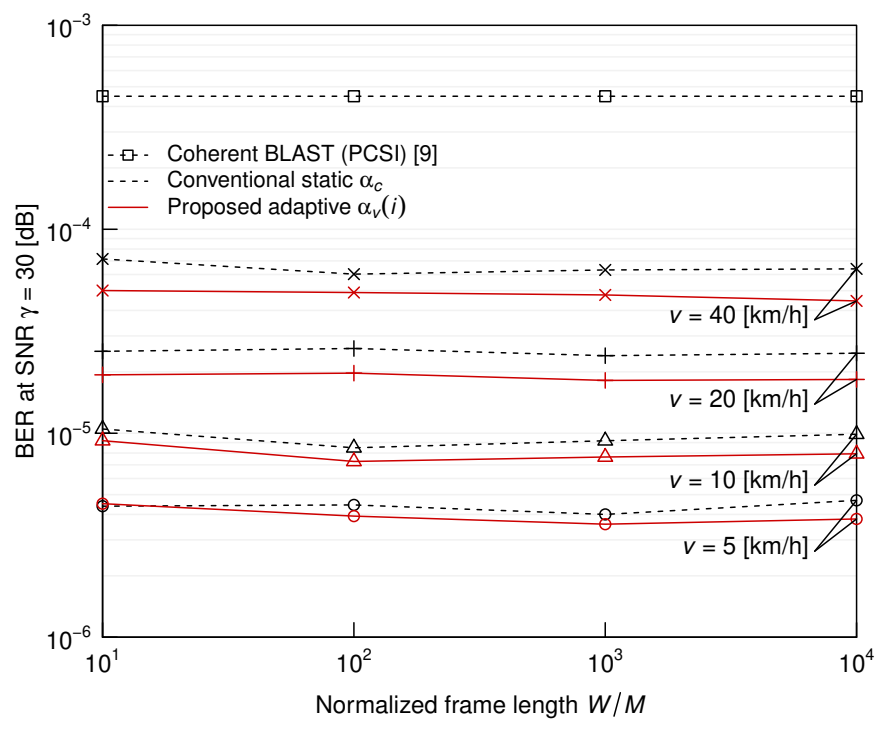

Fig. 5. BER comparisons with respect to the normalized frame length $W / M$, where the SNR was fixed to $\gamma=30[\mathrm{~dB}]$, and we had $(M, N)=(2,2)$. The transmission rate was $R=2[\mathrm{bps} / \mathrm{Hz}]$ and the mobile speed was varied from $v=5$ to $40[\mathrm{~km} / \mathrm{h}]$.

the DUC [41], as well as the ADSM [48] square matrices to nonsquare matrices. Here, we also plotted the MED of the star-QAM [76] constellations having $\mathcal{L}=2,4,8, \cdots, 2^{10}$ for reference. In Rayleigh fading scenarios, the reciprocal of MED, i.e., 1/MED, correlates with the effective SNR that achieves a specific BER [13]. As shown in Fig. 4, the squareto-nonsquare mapping clearly improved both the transmission rate and the MED. Note that it can be expected from this figure that the conventional square schemes achieve lower MED upon increasing the number of transmit subarrays $M$. This implies that the conventional square schemes are not suitable for largescale scenarios.

Fig. 5 shows our BER comparisons for the proposed scheme

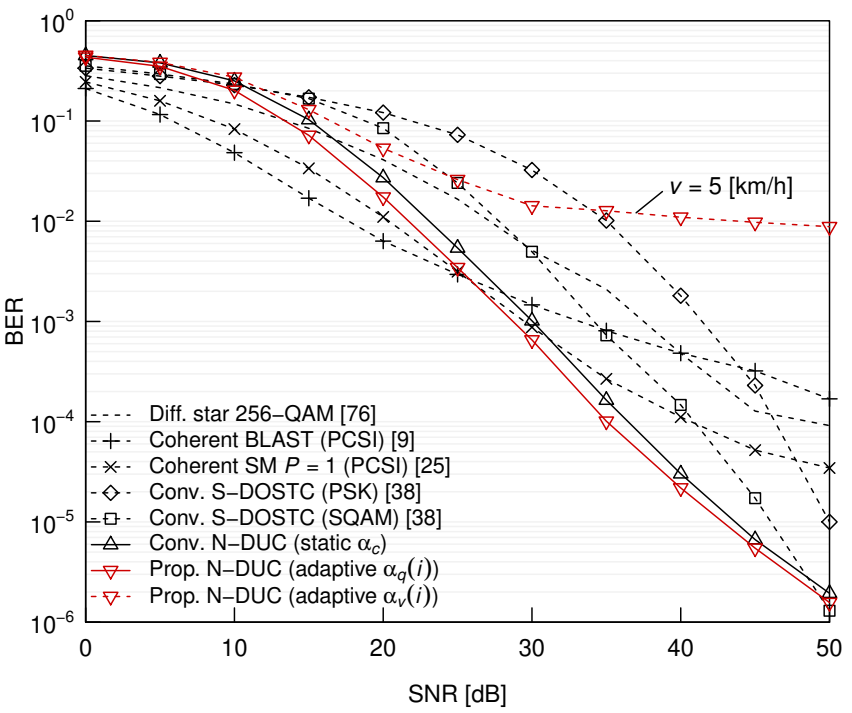

Fig. 6. BER comparisons of the proposed N-DUC and the conventional SDOSTC schemes, where we had $(M, N)=(2,2)$. The transmission rate was $R=8[\mathrm{bps} / \mathrm{Hz}]$ and the mobile speed was set to $v=0[\mathrm{~km} / \mathrm{h}]$.

having the fixed SNR of $\gamma=30[\mathrm{~dB}]$, where the mobile speed was increased from $v=5$ to $40[\mathrm{~km} / \mathrm{h}]$. Additionally, the normalized frame length $W / M$ was varied from $10^{1}$ to $10^{4}$, which corresponded to the reference insertion ratio of $10 \%$ and $0.01 \%$, respectively. Here, we considered the low-rate scenario of $(M, N, R)=(2,2,2)$ in order to obtain the precise average of BER for the normalized frame length of $W / M=10^{4} \%$ scenario, i.e., the pilot insertion ratio of $0.01 \%$, which required $2 \cdot 10^{4} \cdot 10^{7}$-block Monte-Carlo simulations. As shown in Fig. 5, although the conventional forgetting factor $\alpha_{c}$ was found by exhaustive search at each point, the proposed forgetting factor $\alpha_{v}(i)$ dispensing with previous optimization achieved the best BER. Furthermore, the achievable BER remained unchanged upon decreasing the reference insertion ratio. This implies that the performance of the proposed adaptive nonsquare scheme is not dependent on the frame length and on the reference insertion ratio, obeying the same trend as the conventional square DSTC scheme. Based on this observation, we use the pilot insertion ratio of $0.1 \%$ for all the simulation setups.

Fig. 6 shows our BER comparisons between the proposed $\mathrm{N}$-DUC and the conventional S-DOSTC schemes. Here, we considered the $(M, N)=(2,2)$ and $R=8[\mathrm{bps} / \mathrm{Hz}]$ scenario. The mobile speed was set to $v=0[\mathrm{~km} / \mathrm{h}]$. As shown in Fig. 6, our proposed scheme outperformed the coherent SM and BLAST schemes that have PCSI for SNR $\gamma \geq 25$ [dB]. The achievable diversity order of the proposed scheme having $T=1$ is calculated as $N \cdot T=2$. Thus, the conventional S-DOSTC scheme having $T=2$ outperformed the proposed scheme for SNR $\gamma \geq 50$ [dB]. By contrast, in Fig. 6, the proposed scheme exhibited an error floor formation for the $v=5[\mathrm{~km} / \mathrm{h}]$ case, which is a typical walking speed [77]. This implies that the proposed concept is not effective for smallscale scenarios, and it is recommended to use the conventional square schemes instead of the nonsquare schemes.

Similar to Fig. 6, in Fig. 7, we compared the proposed N- 


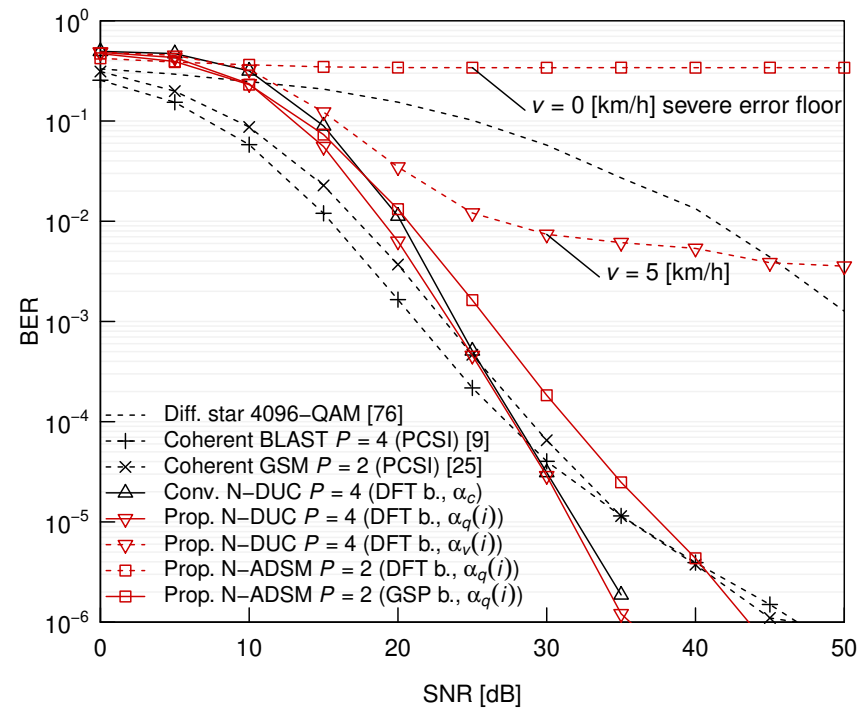

Fig. 7. BER comparisons of the proposed N-DUC and the conventional SDOSTC schemes, where we had $(M, N)=(4,4)$. The transmission rate was $R=12[\mathrm{bps} / \mathrm{Hz}]$ and the mobile speed was set to $v=0[\mathrm{~km} / \mathrm{h}]$.

DUC and the conventional S-DOSTC schemes, where we had $M=4$ transmit subarrays and $N=4$ receive subarrays. The transmission rate was $R=12[\mathrm{bps} / \mathrm{Hz}]$, and the mobile speed was set to $v=0[\mathrm{~km} / \mathrm{h}]$. As shown in Fig. 7 , the N-DUC scheme having the $P=4$ DFT basis and the ADSM scheme having the $P=2$ GSP basis achieved a similar trend to the coherent SM and BLAST schemes. As in Fig. 6, the proposed forgetting factor $\alpha_{q}(i)$ achieved some modest gains against the conventional forgetting factor $\alpha_{c}$, while $\alpha_{q}(i)$ does not require any high-complexity exhaustive search. Note that the ADSM scheme having the DFT basis exhibited severe performance loss, as anticipated in Section IV-A.

Overall, in the small-scale, high-rate and time-varying scenarios, our proposed scheme exhibited error floors, as shown in Figs. 6 and 7. This performance loss was caused by the recursive construction of Eq. (13). Specifically, when the number of receive subarrays is small, $\mathbf{Y}(i)$ in Eq. (13) contains less information concerning the channel coefficients, and $\hat{\mathbf{Y}}(i)$ becomes different from $\mathbf{H}(i) \tilde{\mathbf{S}}(i)$.

\section{B. Large-Scale Scenarios}

Secondly, we conducted $J_{\mathrm{MSE}}$ and BER comparisons for large-scale scenarios, where the numbers of transmit and receive subarrays were increased up to $M=N=16 .{ }^{12}$ Here, the number of antenna elements was as large as $4 \cdot M=48$, which required about $30[\mathrm{~cm}]$ of space. Note that the MED comparison of large-scale scenarios was omitted, because the achievable gains became explicit in Fig. 4.

In Fig. 8, we compared $J_{\mathrm{MSE}}$ of Eq. (16), which has a dominant effect on the achievable BER performance. Here, we considered the conventional static forgetting factor $\alpha_{c}$

\footnotetext{
${ }^{12}$ The largest feasible setup may be $M=18$ because a mmWave channel model has $L=18$ multipath components on average [4]. A larger setup of $M>18$ may not achieve the desired throughput in practical real-world mmWave communications.
}

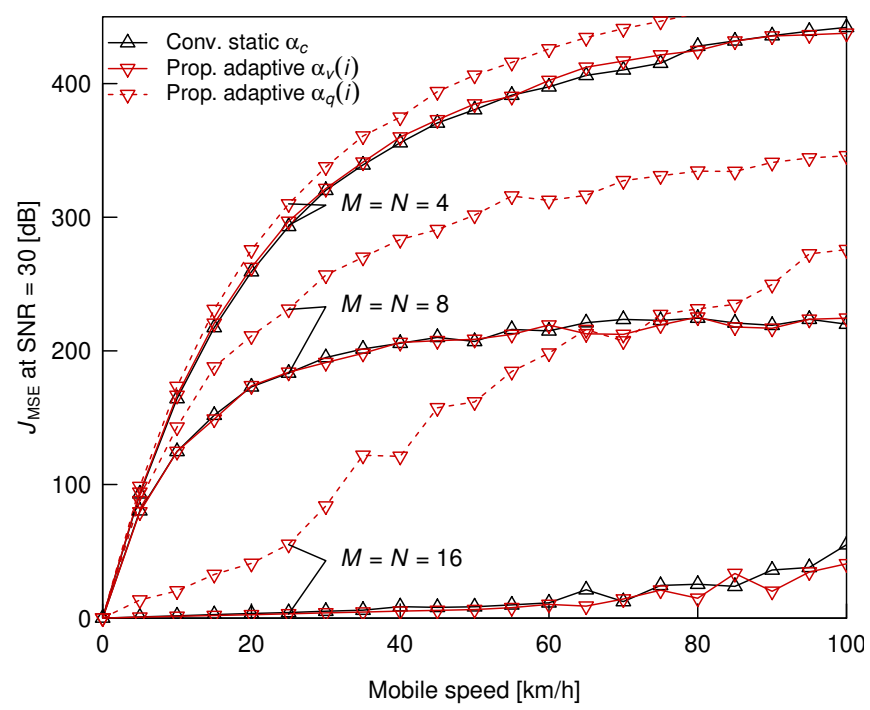

Fig. 8. $J_{\mathrm{MSE}}$ comparisons for the static and adaptive forgetting factors, where the mobile speed was increased from $v=0$ to $100[\mathrm{~km} / \mathrm{h}]$.

of Eq. (15), the proposed adaptive forgetting factors $\alpha_{q}(i)$ of Eq. (29), and $\alpha_{v}(i)$ of Eq. (30). The conventional static forgetting factor $\alpha_{c}$ was designed for minimizing $J_{\mathrm{MSE}}$ for each simulation parameter set, which was a time-consuming task as described in Section III-C. The numbers of transmit and receive subarrays were $M=N=4,8$, and 16 , which were the same as those used in Figs. 7, 9, and 11. As shown in Fig. 8, the static forgetting factor $\alpha_{c}$ achieved the lowest $J_{\mathrm{MSE}}$ at each point, but it required high-complexity optimization. Here, the proposed forgetting factor $\alpha_{v}(i)$ achieved the same $J_{\mathrm{MSE}}$ as $\alpha_{c}$, although $\alpha_{v}(i)$ dose not require any previous optimization. Since the adaptive forgetting factor $\alpha_{q}(i)$ was only designed for quasi-static channels, i.e., for $v=0[\mathrm{~km} / \mathrm{h}]$, it exhibited severe losses for $v>0$ scenarios. It is worth noting that $J_{\text {MSE }}$ significantly improved, as the numbers of transmit and receive subarrays increased. For the $M=N=16$ case, $J_{\mathrm{MSE}}$ was below 10.0 between velocities of $v=0$ and $60[\mathrm{~km} / \mathrm{h}]$. This implies that our proposed scheme is especially beneficial for large-scale scenarios, while the pilot estimation overhead becomes a heavy burden for the conventional coherent MIMO schemes.

Fig. 9 shows our BER comparisons between the proposed $\mathrm{N}$-DUC and the conventional coherent schemes, where the transmission rate was $R=4,8$ and $12[\mathrm{bps} / \mathrm{Hz}]$. The BER curves of the differential star-QAM scheme were plotted for reference. The conventional S-DOSTC scheme could only be considered in Fig. 9(a) due to the complexity issue. As shown in Fig. 9, for the $v=0[\mathrm{~km} / \mathrm{h}]$ case, the proposed $\mathrm{N}$ DUC scheme achieved similar BER to the coherent SM and BLAST schemes. As the SNR increased, the performance gap between the conventional coherent scheme and the proposed scheme decreased. This is because the error $J_{\mathrm{MSE}}$ composed of the noise term $\mathbf{V}(i)$ improves upon increasing the SNR. A comprehensive analysis is provided in [52]. For the $R \geq 8$ [bps $/ \mathrm{Hz}]$ and $v=5[\mathrm{~km} / \mathrm{h}]$ scenarios, the proposed scheme exhibited error floors, while the floors were improved by the 


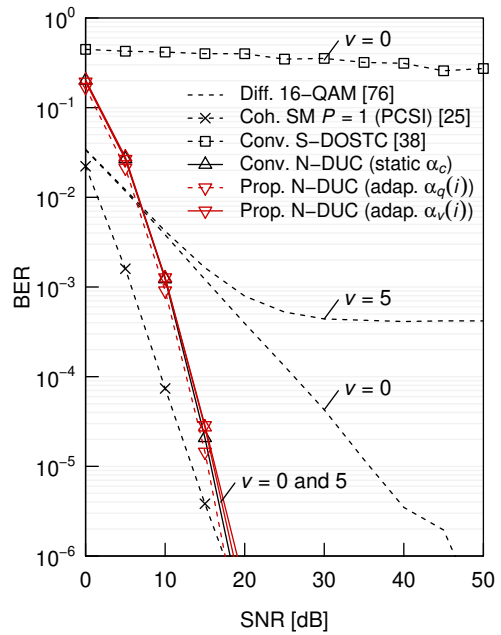

(a) $R=4[\mathrm{bps} / \mathrm{Hz}]$

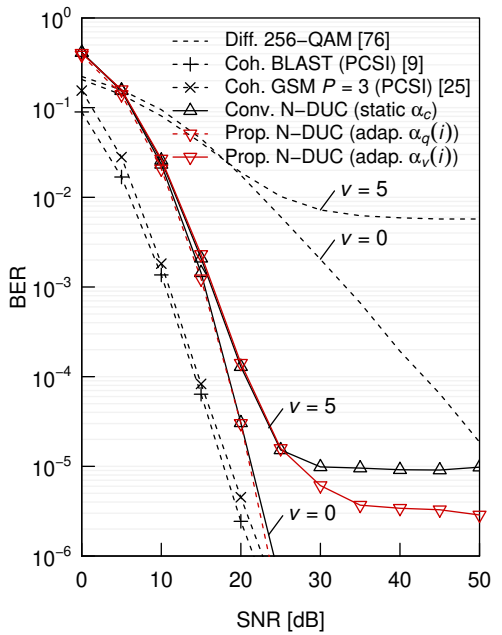

(b) $R=8[\mathrm{bps} / \mathrm{Hz}]$

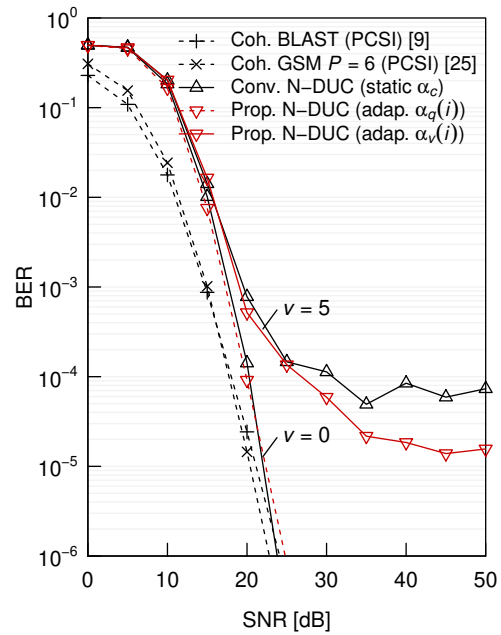

(c) $R=16[\mathrm{bps} / \mathrm{Hz}]$

Fig. 9. BER comparisons of the proposed N-DUC and the conventional S-DOSTC schemes, where we had $(M, N)=(8,8)$. The mobile speed was set to $v=0$ and $5[\mathrm{~km} / \mathrm{h}]$.

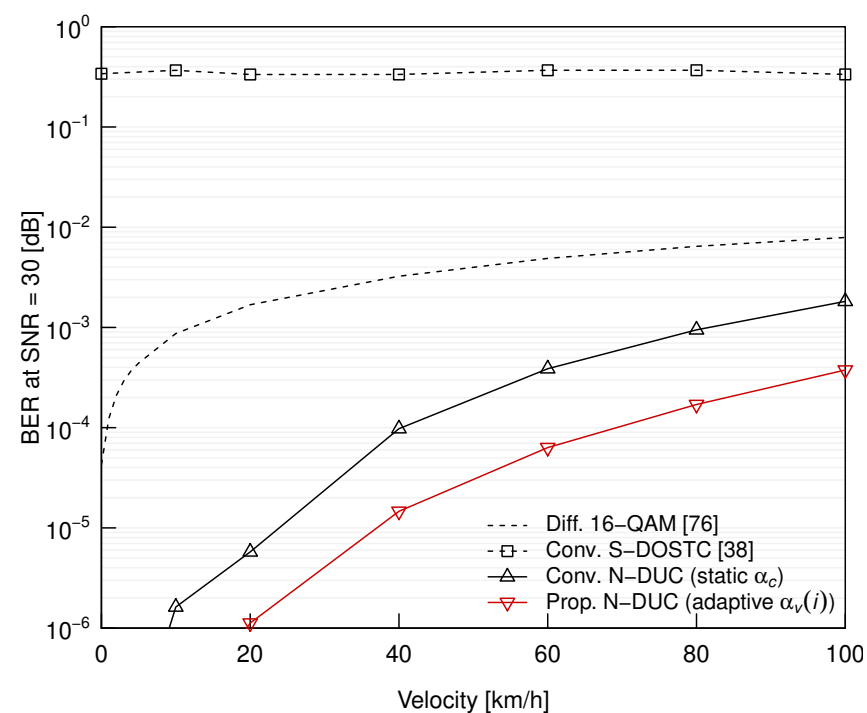

Fig. 10. Error floor comparisons for the proposed scheme, where the simulation parameters were the same as those used in Fig. 9(a).

proposed adaptive forgetting factor $\alpha_{v}(i)$.

In order to investigate the error-floor-mitigation effect of $\alpha_{v}(i)$, as observed in Fig. 9, we varied the mobile speed from $v=0$ to $100[\mathrm{~km} / \mathrm{h}]$ in Fig. 10. Here, the BER at $\mathrm{SNR}=30[\mathrm{~dB}]$ was calculated for each scheme. As shown in Fig. 10, the proposed forgetting factor $\alpha_{v}(i)$ outperformed the conventional $\alpha_{c}$ across the entire region. Thus, the proposed $\alpha_{v}(i)$ is beneficial for high-mobility scenarios.

According to Fig. 8, the error $J_{\mathrm{MSE}}$ can be mitigated by increasing the numbers of transmit and receive subarrays. Then, we considered the $M=N=16$ setup in Fig. 11, where the mobile speed was increased from $v=0$ to $40[\mathrm{~km} / \mathrm{h}]$. The transmission rate was $R=16[\mathrm{bps} / \mathrm{Hz}]$ for the BPSK-aided BLAST scheme, $R=12$ [bps/Hz] for the BPSK-aided GSM scheme, and $R=11[\mathrm{bps} / \mathrm{Hz}]$ for both the N-DUC and NADSM schemes, where those different rates were compared

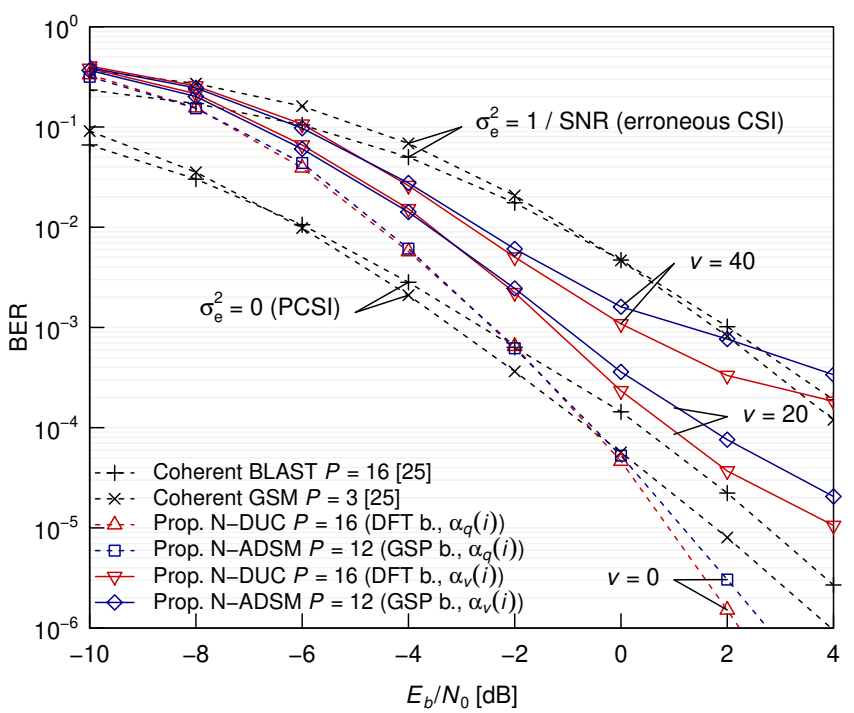

Fig. 11. BER comparisons of the proposed N-DUC and the conventional SDOSTC schemes, where we had $(M, N)=(16,16)$. The mobile speed was increased from $v=0$ to $40[\mathrm{~km} / \mathrm{h}]$. The transmission rates were $R=11,12$, and 16 , which were appropriate by compared by considering $E_{b} / N_{0}$.

vs. $E_{b} / N_{0}$ instead of SNR. In Fig. 11, we additionally considered the channel estimation errors of $\mathcal{C N}(0,1 / \mathrm{SNR})$ [78]. As shown in Fig. 11, for the $v=0[\mathrm{~km} / \mathrm{h}]$ and $E_{b} / N_{0} \geq 0[\mathrm{~dB}]$ case, the proposed schemes outperformed the coherent BLAST and GSM schemes that have PCSI. Here, the performance of the N-ADSM scheme approached that of the N-DUC scheme, while the detection complexity was reduced by using the $P=12$ GSP basis. Additionally, for the $v=40[\mathrm{~km} / \mathrm{h}]$ case, the proposed schemes outperformed the coherent schemes below $E_{b} / N_{0} \leq 2$ [dB], provided that the channel estimation errors existed. This implies that our proposed scheme is capable achieving performance gains below $v=40[\mathrm{~km} / \mathrm{h}]$, while eliminating the channel estimation overhead. Thus, the proposed scheme is especially beneficial 
for large-scale high-mobility scenarios.

\section{CONCLUSiOnS}

In this paper, we proposed the novel GSP-aided basis set, which imposes no limitation on the number of nonzero elements $N_{b}$ and achieves a high coding gain over the conventional DFT basis set. Then, we also proposed a pair of adaptive forgetting factors for the general nonsquare DSTC scheme, that support the general time-invariant and time-varying channels, respectively. The proposed differential mmWave system only relies on ABF both at transmitter and receiver, and does not require the complex-valued channel coefficient feedback, while its frame length becomes longer as the numbers of subarrays and subcarriers increase. In our simulations, the proposed nonsquare scheme achieved similar performances to the classic BLAST and GSM schemes having PCSI. Specifically, the performance of the proposed N-ADSM scheme having the GSP basis was capable of approaching that of the N-DUC scheme having the conventional DFT basis, while reducing the detection complexity. Additionally, the proposed adaptive forgetting factor dispensing with any previous optimization outperformed the conventional static forgetting factor that required time-consuming exhaustive search.

The key feature of the proposed scheme is its high reliability in large-scale scenarios. The BER performance improves upon increasing the number of subarrays, although the channel estimation overhead escalates with the system's scale. Furthermore, our proposed scheme also performed better than the ideal coherent MIMO schemes for $v \leq 40[\mathrm{~km} / \mathrm{h}]$ scenarios. Based on these observations, we conclude that the proposed large-scale differential GSM system is capable of reliable operation in high-mobility mmWave channels.

\section{APPENDIX A}

THE GSP BASIS SETS FOR ADSM

For the $(M, O, L)=(4,2,16)$ and $N_{b}=2$ case, we used the following basis:

$$
\mathbf{E}_{1}=[0,-0.753584+0.112447 \jmath, 0, \quad-0.218968-0.609525 \jmath]^{\mathrm{H}},
$$

which achieved the MED of 0.0638601. Additionally, for the $(M, O, L)=(16,2,4)$ and $N_{b}=12$ case, we used the following basis:

$$
\begin{aligned}
\mathbf{E}_{1}= & {[0.415493+0.200765 \jmath, 0.0319892-0.0912603 \jmath,} \\
& -0.10222+0.0535648 \jmath, 0.102279+0.0610215 \jmath, \\
& 0.259023+0.410754 \jmath,-0.0230858+0.00884931 \jmath, \\
& -0.0521544-0.115707 \jmath, 0,0.115496-0.0806238 \jmath, \\
& 0.295856-0.044537 \jmath, 0.377234+0.373951 \jmath, \\
& -0.0332786-0.30913 \jmath, 0,-0.0848214+0.0480732 \jmath, 0,0]^{\mathrm{H}},
\end{aligned}
$$

which achieved the MED of 0.877168 .

\section{APPENDIX B}

\section{The Designed Constellation Factors for DUC}

The DUC codewords are designed so as to maximize the diversity product [41]. In this Appendix, we use the same mathematical symbols as [41], such as the number of TAs $M$, the number of codewords $L$, the constellation design factors $\left[\begin{array}{lll}u_{1} & \cdots & u_{M}\end{array}\right]$, and the diversity product $\xi$.
In Fig. 6, we used the factors of $\left[\begin{array}{ll}u_{1} & u_{2}\end{array}\right]=\left[\begin{array}{ll}1 & 75\end{array}\right]$ for the $(M, L)=(2,256)$ case, which achieved the diversity product of $\xi=0.3143625$.

In Fig. 7, we used [1 5751059 1921] for the $(M, L)=$ $(4,4096)$ case, which achieved $\xi=0.5672944$.

In Fig. 9, we considered $M=8$ and used $\left[\begin{array}{lllllll}1 & 5 & 5 & 5 & 5 & 5 & 6\end{array}\right]$

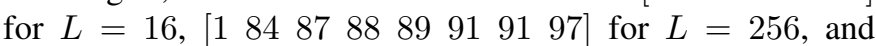
[1 167221701420852223212378124192 29994] for $L=$ 65536 , each of which achieved $\xi=0.7168117,0.5197687$, and 0.2244139 , respectively.

In Fig. 11, we used [1 446516555609640644694712718 $723724724772787805]$ for the $(M, L)=(16,2048)$ case, which achieved $\xi=0.5681189$.

\section{REFERENCES}

[1] R. Daniels, J. Murdock, T. Rappaport, and R. W. Heath, "60 GHz wireless: Up close and personal," IEEE Microwave Magazine, vol. 11, no. 7, pp. 44-50, 2010

[2] T. S. Rappaport, R. Mayzus, Y. Azar, K. Wang, G. N. Wong, J. K. Schulz, M. Samimi, and F. Gutierrez, "Millimeter wave mobile communications for 5G cellular: it will work!" IEEE Access, vol. 1, pp. $335-349,2013$.

[3] S. Rangan, T. S. Rappaport, and E. Erkip, "Millimeter-wave cellular wireless networks: Potentials and challenges," Proceedings of the IEEE, vol. 102, no. 3, pp. 366-385, 2014.

[4] I. A. Hemadeh, K. Satyanarayana, M. El-Hajjar, and L. Hanzo, "Millimeter-wave communications: Physical channel models, design considerations, antenna constructions and link-budget," IEEE Соттиnications Surveys and Tutorials, vol. 20, no. 2, pp. 870-913, 2018.

[5] A. Alkhateeb, O. El Ayach, G. Leus, and R. W. Heath, "Hybrid precoding for millimeter wave cellular systems with partial channel knowledge," in Information Theory and Applications Workshop, San Diego, CA, USA, Feb. 10-15, 2013.

[6] R. Rajashekar and L. Hanzo, "Iterative matrix decomposition aided block diagonalization for mm-Wave multiuser MIMO systems," IEEE Transactions on Wireless Communications, vol. 16, no. 3, pp. 13721384, 2017.

[7] E. Perahia, C. Cordeiro, M. Park, and L. L. Yang, "IEEE 802.11ad: Defining the next generation," in IEEE Consumer Communications and Networking Conference, Las Vegas, NV, USA, Feb. 25, 2010.

[8] T. Nitsche, C. Cordeiro, A. B. Flores, E. W. Knightly, E. Perahia, and J. C. Widmer, "IEEE 802.11ad: Directional $60 \mathrm{GHz}$ communication for multi-gigabit-per-second Wi-Fi," IEEE Communications Magazine, vol. 52, no. 12, pp. 132-141, 2014.

[9] P. Wolniansky, G. Foschini, G. Golden, and R. Valenzuela, "V-BLAST: an architecture for realizing very high data rates over the rich-scattering wireless channel," in Proceedings of the International Symposium on Signals, Systems, and Electronics, Pisa, Italy, Oct. 2, 1998.

[10] Y. Ghasempour, C. R. C. M. Silva, C. Cordeiro, and E. W. Knightly, "IEEE 802.11ay: Next-generation $60 \mathrm{GHz}$ communication for $100 \mathrm{~Gb} / \mathrm{s}$ Wi-Fi," IEEE Communications Magazine, vol. 55, no. 12, pp. 186-192, 2017.

[11] E. Basar, M. Wen, R. Mesleh, M. Di Renzo, Y. Xiao, and H. Haas, "Index modulation techniques for next-generation wireless networks," IEEE Access, vol. 5, pp. 16693-16746, 2017.

[12] M. Wen, X. Cheng, and L. Yang, Index modulation for $5 G$ wireless communications. Springer, 2017.

[13] N. Ishikawa, S. Sugiura, and L. Hanzo, "50 years of permutation, spatial and index modulation: From classic RF to visible light communications and data storage," IEEE Communications Surveys and Tutorials, vol. 20, no. 3, pp. 1905-1938, 2018.

[14] P. Yang, Y. Xiao, Y. L. Guan, K. V. Hari, A. Chockalingam, S. Sugiura, H. Haas, M. Di Renzo, C. Masouros, Z. Liu, L. Xiao, S. Li, and L. Hanzo, "Single-carrier SM-MIMO: A promising design for broadband large-scale antenna systems," IEEE Communications Surveys \& Tutorials, vol. 18, no. 3, pp. 1687-1716, 2016.

[15] P. Liu and A. Springer, "Space shift keying for LOS communication at mmWave frequencies," IEEE Wireless Communications Letters, vol. 4, no. 2, pp. 121-124, 2015

[16] P. Liu, M. Di Renzo, and A. Springer, "Line-of-sight spatial modulation for indoor mmWave communication at $60 \mathrm{GHz}$," IEEE Transactions on Wireless Communications, vol. 15, no. 11, pp. 7373-7389, 2016. 
[17] R. Mesleh and A. Younis, "Capacity analysis for LOS millimeter-wave quadrature spatial modulation," Wireless Networks, pp. 1-10, 2017.

[18] N. S. Perovic, P. Liu, M. D. Renzo, and A. Springer, "Receive spatial modulation for LOS mmWave communications based on TX beamforming," IEEE Communications Letters, vol. 21, no. 4, pp. 1089-7798, 2017.

[19] Y. Ding, K. J. Kim, T. Koike-Akino, M. Pajovic, P. Wang, and P. Orlik, "Spatial scattering modulation for uplink millimeter-wave systems," IEEE Communications Letters, vol. 21, no. 7, pp. 1493-1496, 2017.

[20] I. A. Hemadeh, M. El-Hajjar, S. Won, and L. Hanzo, "Layered multigroup steered space-time shift-keying for millimeter-wave communications," IEEE Access, vol. 4, pp. 3708-3718, 2016.

[21] I. A. Hemadeh, P. Botsinis, M. El-Hajjar, S. Won, and L. Hanzo, "Reduced-RF-chain aided soft-decision multi-set steered space-time shift-keying for millimeter-wave communications," IEEE Access, vol. 5, pp. 7223-7243, 2017.

[22] C. Sacchi, T. Rahman, I. A. Hemadeh, and M. El-Hajjar, "Millimeterwave transmission for small-cell backhaul in dense urban environment: a solution based on MIMO-OFDM and space-time shift keying (STSK),' IEEE Access, vol. 5, pp. 4000-4017, 2017.

[23] P. Botsinis, I. Hemadeh, D. Alanis, Z. Babar, H. Nguyen, D. Chandra, S. X. Ng, M. El-Hajjar, and L. Hanzo, "Joint-alphabet space time shift keying in mm-wave non-orthogonal multiple access," IEEE Access, vol. 6, pp. 22 602-22 621, 2017.

[24] I. A. Hemadeh, S. Lu, M. El-Hajjar, and L. Hanzo, "Compressed sensing-aided index modulation improves space-time shift keying assisted millimeter-wave communications," IEEE Access, in press.

[25] N. Ishikawa, R. Rajashekar, S. Sugiura, and L. Hanzo, "Generalizedspatial-modulation-based reduced-RF-chain millimeter-wave communications," IEEE Transactions on Vehicular Technology, vol. 66, no. 1, pp. 879-883, 2017.

[26] N. S. Perovic, P. Liu, and A. Springer, "Design of a simple phase precoder for generalized spatial modulation in LOS millimeter wave channels," in International ITG Conference on Systems, Communications and Coding, Hamburg, Germany, Feb. 6-9, 2017.

[27] L. He, J. Wang, and J. Song, "Spectral-efficient analog precoding for generalized spatial modulation aided mmWave MIMO," IEEE Transactions on Vehicular Technology, vol. 66, no. 10, pp. 9598-9602, 2017.

[28] — "Spatial modulation for more spatial multiplexing: RF-chainlimited generalized spatial modulation aided mm-wave MIMO with hybrid precoding," IEEE Transactions on Communications, vol. 66, no. 3, pp. 986-998, 2018

[29] P. Liu, M. Di Renzo, and A. Springer, "Variable-Nu generalized spatial modulation for indoor los mmwave communication: Performance optimization and novel switching structure," IEEE Transactions on Communications, vol. 65, no. 6, pp. 2625-2640, 2017.

[30] P. Liu, J. Blumenstein, N. S. Perovic, M. Di Renzo, and A. Springer, "Performance of generalized spatial modulation MIMO over measured 60GHz indoor channels," IEEE Transactions on Communications, vol. 66, no. 1, pp. 133-148, 2018.

[31] J. Jeganathan, A. Ghrayeb, and L. Szczecinski, "Generalized space shift keying modulation for MIMO channels," in IEEE International Symposium on Personal, Indoor and Mobile Radio Communications, Cannes, France, Sept. 15-18, 2008.

[32] S. A. Busari, K. M. S. Huq, S. Mumtaz, L. Dai, and J. Rodriguez, "Millimeter-wave massive MIMO communication for future wireless systems: A survey," IEEE Communications Surveys \& Tutorials, in press, vol. 20, no. 2, pp. 836-869, 2017.

[33] A. L. Swindlehurst, E. Ayanoglu, P. Heydari, and F. Capolino, "Millimeter-wave massive MIMO: the next wireless revolution?" IEEE Communications Magazine, vol. 52, no. 9, pp. 56-62, 2014.

[34] P. Marinier, G. Y. Delisle, and C. L. Despins, "Temporal variations of the indoor wireless millimeter-wave channel," IEEE Transactions on Antennas and Propagation, vol. 46, no. 6, pp. 928-934, 1998.

[35] G. R. MacCartney, T. S. Rappaport, S. Sun, and S. Deng, "Indoor office wideband millimeter-wave propagation measurements and channel models at 28 and $73 \mathrm{GHz}$ for Ultra-Dense 5G Wireless Networks," IEEE Access, vol. 3, pp. 2388-2424, 2015.

[36] S. Geng, J. Kivinen, X. Zhao, and P. Vainikainen, "Millimeter-wave propagation channel characterization for short-range wireless communications," IEEE Transactions on Vehicular Technology, vol. 58, no. 1 , pp. 3-13, 2009.

[37] Z. Xiao, X. G. Xia, D. Jin, and N. Ge, "Iterative eigenvalue decomposition and multipath-grouping $\mathrm{Tx} / \mathrm{Rx}$ joint beamformings for millimeterwave communications," IEEE Transactions on Wireless Communications, vol. 14, no. 3, pp. 1595-1607, 2015.
[38] V. Tarokh and H. Jafarkhani, "A differential detection scheme for transmit diversity," IEEE Journal on Selected Areas in Communications, vol. 18, no. 7, pp. 1169-1174, 2000.

[39] B. M. Hochwald and T. L. Marzetta, "Unitary space-time modulation for multiple-antenna communications in Rayleigh flat fading," IEEE Transactions on Information Theory, vol. 46, no. 2, pp. 543-564, 2000.

[40] B. Hassibi and B. Hochwald, "Cayley differential unitary space-time codes," IEEE Transactions on Information Theory, vol. 48, no. 6, pp. 1485-1503, 2002.

[41] B. M. Hochwald and W. Sweldens, "Differential unitary space-time modulation," IEEE Transactions on Communications, vol. 48, no. 12, pp. 2041-2052, 2000

[42] Y. Bian, M. Wen, X. Cheng, H. Poor, and B. Jiao, "A differential scheme for spatial modulation," in IEEE Global Communications Conference, Atlanta, GA, USA, Dec. 9-13, 2013.

[43] Y. Bian, X. Cheng, M. Wen, L. Yang, H. V. Poor, and B. Jiao, "Differential spatial modulation," IEEE Transactions on Vehicular Technology, vol. 64 , no. 7 , pp. 3262-3268, 2015.

[44] N. Ishikawa and S. Sugiura, "Unified differential spatial modulation," IEEE Wireless Communications Letters, vol. 3, no. 4, pp. 337-340, 2014.

[45] C. Xu, R. Rajashekar, N. Ishikawa, S. Sugiura, and L. Hanzo, "SingleRF index shift keying aided differential space-time block coding," IEEE Transactions on Signal Processing, vol. 66, no. 3, pp. 773-788, 2018.

[46] C. Xu, P. Zhang, R. Rajashekar, N. Ishikawa, S. Sugiura, L. Wang, and L. Hanzo, "Finite-cardinality single-RF differential space-time modulation for improving the diversity-throughput tradeoff," IEEE Transactions on Communications, in press, vol. 67, no. 1, pp. 318-335, 2019.

[47] R. Rajashekar, N. Ishikawa, S. Sugiura, K. V. S. Hari, and L. Hanzo, "Full-diversity dispersion matrices from algebraic field extensions for differential spatial modulation," IEEE Transactions on Vehicular Technology, vol. 66, no. 1, pp. 385-394, 2017.

[48] R. Rajashekar, C. Xu, N. Ishikawa, S. Sugiura, K. V. S. Hari, and L. Hanzo, "Algebraic differential spatial modulation is capable of approaching the performance of its coherent counterpart," IEEE Transactions on Communications, vol. 65, no. 10, pp. 4260-4273, 2017.

[49] C. Xu, J. Zhang, T. Bai, S. P. Botsinis, R. G. Maunder, R. Zhang, and L. Hanzo, "Adaptive coherent/non-coherent single/multiple-antenna aided channel coded ground-to-air aeronautical communication," IEEE Transactions on Communications, in press.

[50] R. Y. Mesleh, H. Haas, S. Sinanovic, C. Ahn, and S. Yun, "Spatia modulation," IEEE Transactions on Vehicular Technology, vol. 57, no. 4, pp. 2228-2241, 2008 .

[51] N. Ishikawa and S. Sugiura, "Rectangular differential spatial modulation for open-loop noncoherent massive-MIMO downlink," IEEE Transactions on Wireless Communications, vol. 16, no. 3, pp. 1908-1920, 2017.

[52] N. Ishikawa, R. Rajashekar, C. Xu, S. Sugiura, and L. Hanzo, "Differential space-time coding dispensing with channel-estimation approaches the performance of its coherent counterpart in the open-loop massive MIMO-OFDM downlink," IEEE Transactions on Communications, vol. 66, no. 12, pp. 6190-6204, 2018.

[53] C. Wu, Y. Xiao, L. Xiao, P. Yang, and X. Lei, "Space-time block coded rectangular differential spatial modulation," in IEEE International Conference on Communications, Kansas City, MO, USA, May 20-24, 2018.

[54] L. Xiao, P. Xiao, Y. Xiao, C. Wu, D. Mi, and I. A. Hemadeh, "Rectangular differential OFDM with index modulation," in IEEE Vehicular Technology Conference, Kuala Lumpur, Malaysia, Apr. 28 - May 1, 2019.

[55] D. K. Kahaner, "HDTV research in Japan," IEEE Micro, vol. 13, no. 5, pp. 49-53, 1993.

[56] R. Heath and A. Paulraj, "Linear dispersion codes for MIMO systems based on frame theory," IEEE Transactions on Signal Processing, vol. 50, no. 10, pp. 2429-2441, 2002.

[57] IEEE Standards Association, IEEE Standard for information technology-telecommunications and information exchange between systems local and metropolitan area networks-specific requirements - part 11: Wireless LAN medium access control (MAC) and physical layer (PHY) specifications. IEEE, 2016.

[58] C. Cordeiro, D. Akhmetov, and M. Park, "IEEE 802.11ad: Introduction and performance evaluation of the first multi-Gbps WiFi technology," in Proceedings of the ACM international workshop on mmWave communications, Chicago, Illinois, USA, Sept. 24, 2010.

[59] J. Rinne and M. Renfors, "Pilot spacing in orthogonal frequency division multiplexing systems on practical channels," IEEE Transactions on Consumer Electronics, vol. 42, no. 4, pp. 959-962, 1996. 
[60] B. Ye and Z. Zhang, "Improved pilot design and channel estimation for 60GHz OFDM based on IEEE 802.11.ad," in IEEE Wireless Communications and Networking Conference, Shanghai, China, Apr.7-10, 2013.

[61] L. Hanzo, Y. Akhtman, L. Wang, and M. Jiang, MIMO-OFDM for LTE, WIFI and WIMAX. Wiley-IEEE Press, 2010.

[62] O. E. Ayach, S. Rajagopal, S. Abu-Surra, Z. Pi, and R. W. Heath, "Spatially sparse precoding in millimeter wave MIMO systems," IEEE Transactions on Wireless Communications, vol. 13, no. 3, pp. 14991513, 2014.

[63] J. H. Yoo, J. Bae, S. H. Lim, S. Kim, J. W. Choi, and B. Shim, "Sampling-based tracking of time-varying channels for millimeter waveband communications," in IEEE International Conference on Communications, Paris, France, May 21-25, 2017.

[64] T. G. Kolda and B. W. Bader, "Tensor decompositions and applications," SIAM Review, vol. 51, no. 3, pp. 455-500, 2009.

[65] E. Torkildson, U. Madhow, and M. Rodwell, "Indoor millimeter wave MIMO: Feasibility and performance," IEEE Transactions on Wireless Communications, vol. 10, no. 12, pp. 4150-4160, 2011.

[66] Y. Yao and G. Giannakis, "Blind carrier frequency offset estimation in SISO, MIMO, and multiuser OFDM systems," IEEE Transactions on Communications, vol. 53, no. 1, pp. 173-183, 2005.

[67] K. E. Baddour and N. C. Beaulieu, "Autoregressive modeling for fading channel simulation," IEEE Transactions on Wireless Communications, vol. 4, no. 4, pp. 1650-1662, 2005.

[68] T. S. Rappaport, Wireless Communications: Principles and Practice. Prentice Hall, 2002

[69] M. Bhatnagar, A. Hjorungnes, and L. S. L. Song, "Differential coding for non-orthogonal space-time block codes with non-unitary constellations over arbitrarily correlated rayleigh channels," IEEE Transactions on Wireless Communications, vol. 8, no. 8, pp. 3985-3995, 2009.

[70] S. Alamouti, "A simple transmit diversity technique for wireless communications," IEEE Journal on Selected Areas in Communications, vol. 16, no. 8, pp. 1451-1458, 1998.

[71] D. E. Knuth, "Big Omicron and big Omega and big Theta," $A C M$ SIGACT News, vol. 8, no. 2, pp. 18-24, 1976.

[72] V. Tarokh, N. Seshadri, and A. Calderbank, "Space-time codes for high data rate wireless communication: performance criterion and code construction," IEEE Transactions on Information Theory, vol. 44, no. 2, pp. 744-765, 1998.

[73] S. Sugiura, S. Chen, and L. Hanzo, "Coherent and differential spacetime shift keying: a dispersion matrix approach," IEEE Transactions on Communications, vol. 58, no. 11, pp. 3219-3230, 2010.

[74] M. Wen, Y. Zhang, J. Li, E. Basar, and F. Chen, "Equiprobable subcarrier activation method for OFDM with index modulation," IEEE Communications Letters, vol. 20, no. 12, pp. 2386-2389, 2016.

[75] R. Mesleh, H. Haas, C. W. Ahn, and S. Yun, "Spatial modulation - A new low complexity spectral efficiency enhancing technique," in International Conference on Communications and Networking in China, Beijing, China, Oct. 25-27, 2006.

[76] W. Webb, L. Hanzo, and R. Steele, "Bandwidth efficient QAM schemes for Rayleigh fading channels," IEE Proceedings, vol. 138, no. 3, pp. $169-175,1991$.

[77] R. C. Browning, E. A. Baker, J. A. Herron, and R. Kram, "Effects of obesity and sex on the energetic cost and preferred speed of walking," Journal of Applied Physiology, vol. 100, no. 2, pp. 390-398, 2006.

[78] P. Yang, B. Zhang, Y. Xiao, B. Dong, S. Li, M. El-Hajjar, and L. Hanzo, "Detect-and-forward relaying aided cooperative spatial modulation for wireless networks," IEEE Transactions on Communications, vol. 61, no. 11 , pp. $4500-4511,2013$

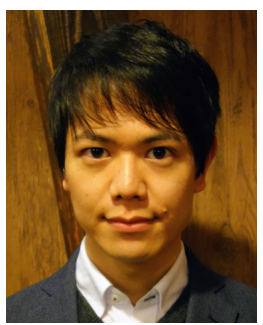

Naoki Ishikawa (S'13-M'17) was born in Kanagawa, Japan, in 1991. He received the B.E., M.E., and $\mathrm{Ph} . \mathrm{D}$. degrees from the Tokyo University of Agriculture and Technology, Tokyo, Japan, in 2014, 2015, and 2017, respectively. From June 2015 to September 2015, he was an academic visitor with the School of Electronics and Computer Science, University of Southampton, UK. From April 2016 to March 2017, he was a research fellow of the Japan Society for the Promotion of Science. From April 2017, he has been an assistant professor in the Graduate School of Information Sciences, Hiroshima City University, Japan.

He was certified as an Exemplary Reviewer of IEEE TRANSACTIONS ON COMMUNICATIONS 2017. He also received eight domestic awards, including the Yasujiro Niwa Outstanding Paper Award from Tokyo Denki University in 2018, the Telecom System Technology student Award (honorable mention) from Telecommunications Advancement Foundation of Japan in 2014, and the Outstanding Paper Award for Young C\&C Researchers from NEC C\&C Foundation in 2014.

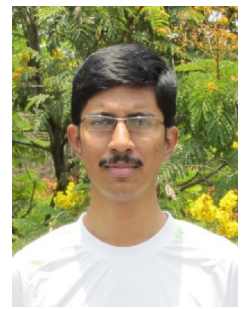

Rakshith Rajashekar (M'14-SM'17) received the B.E. degree in electrical communication engineering from Visvesvaraya Technological University, Karnataka, India, in 2007. He received his Ph.D. from the Department of Electrical Communication Engineering, Indian Institute of Science (IISc), India, in 2014. He is presently working as a Research Fellow at the University of Southampton, UK. Before joining the University of Southampton, he worked at Accord Software \& Systems, Bengaluru, India, as a Systems Engineer from 2007 to 2009, and as a Senior Scientist at Broadcom Communications, Bengaluru, India from 2014 to 2015. His research interests include antenna selection in MIMO systems, differential communication, millimeter wave communication, communication between drones with a focus on space-time signal processing and coding. $\mathrm{He}$ is the recipient of the Special Recognition award from Broadcom Communications, India and the Dean's Award from the University of Southampton, UK, for excellence in research. He has received the Best Reviewer award from IEEE Transactions on Wireless Communications, IEEE Transactions on Communications and IEEE Wireless Communications Letters.

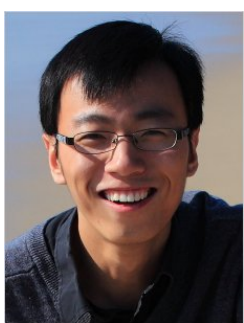

Chao Xu (S'09-M'14) received a B.Eng. degree from Beijing University of Posts and Telecommunications, China, and a BSc(Eng) with First Class Honours from Queen Mary, University of London, UK, through a Sino-UK joint degree program in 2008, both in Telecommunications Enginneering with Management. He obtained a MSc degree with distinction in Radio Frequency Communication Systems and a Ph.D. degree in Wireless Communications from the University of Southampton, UK in 2009 and 2015, respectively. He is currently a postdoctoral researcher working at Southampton Wireless Group, University of Southampton, UK. His research interests include reduced-complexity MIMO design, noncoherent detection, extrinsic-information-transfer-chart-aided turbo detection, and cooperative communications. He was awarded the Best M.Sc. Student in Broadband and Mobile Communication Networks by the IEEE Communications Society (United Kingdom and Republic of Ireland Chapter) in 2009. He also received 2012 Chinese Government Award for Outstanding Self-Financed Student Abroad. 


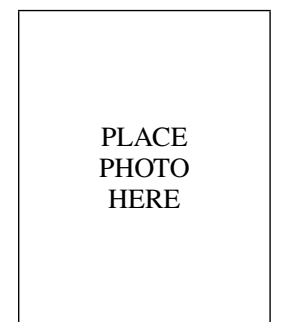

Mohammed El-Hajjar received the Ph.D. degree in wireless communications from the University of Southampton, U.K., in 2008. Following the Ph.D. degree, he joined Imagination Technologies as a Design Engineer, where he worked on designing and developing imagination's multi-standard communications platform, which resulted in three patents. $\mathrm{He}$ is currently an Associate Professor with the Department of Electronics and Computer Science, University of Southampton. His research interests include the design of intelligent and energy-efficient transceivers, cross-layer optimization for large-scale networks, MIMO, millimeter-wave communications, and radio over fiber network design. He was a recipient of several academic awards and has published a Wiley-IEEE book and in excess of 80 journal and conference papers.

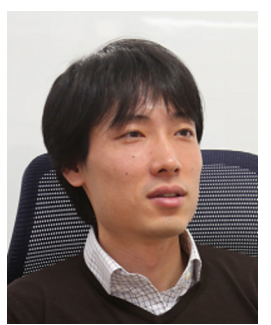

Shinya Sugiura (M'06-SM'12) received the B.S. and M.S. degrees in aeronautics and astronautics from Kyoto University, Kyoto, Japan, in 2002 and 2004, respectively, and the $\mathrm{Ph} . \mathrm{D}$. degree in electronics and electrical engineering from the University of Southampton, Southampton, U.K., in 2010.

From 2004 to 2012, he was a Research Scientist with Toyota Central Research and Development Laboratories, Inc., Aichi, Japan. From 2013 to 2018, he was an Associate Professor with the Department of Computer and Information Sciences, Tokyo University of Agriculture and Technology, Tokyo, Japan. Since 2018, he has been an Associate Professor with the Institute of Industrial Science, University of Tokyo, Tokyo, Japan, where he heads the Wireless Communications Research Group. His research has covered a range of areas in wireless communications, networking, signal processing, and antenna technology. $\mathrm{He}$ authored or coauthored over 60 IEEE journal papers.

Dr. Sugiura was a recipient of a number of awards, including the Sixth RIEC Award from the Foundation for the Promotion of Electrical Communication in 2016, the Young Scientists' Prize by the Minister of Education, Culture, Sports, Science and Technology of Japan in 2016, the 14th Funai Information Technology Award (First Prize) from the Funai Foundation in 2015, the 28th Telecom System Technology Award from the Telecommunications Advancement Foundation in 2013, the Sixth IEEE Communications Society Asia-Pacific Outstanding Young Researcher Award in 2011, the 13th Ericsson Young Scientist Award in 2011, and the 2008 IEEE Antennas and Propagation Society Japan Chapter Young Engineer Award. He was also certified as an Exemplary Reviewer of IEEE COMMUNICATIONS LETTERS in 2013 and 2014, and that of IEEE TRANSACTIONS ON COMMUNICATIONS in 2018.

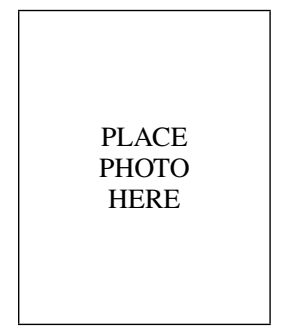

Lie-Liang Yang (M'98-SM'02-F'16) received the B.Eng. degree in communications engineering from Shanghai Tiedao University, Shanghai, China, in 1988, and the M.Eng. and Ph.D. degrees in communications and electronics from Beijing Jiaotong University, Beijing, China, in 1991 and 1997, respectively. In 1997, he was a Visiting Scientist with the Institute of Radio Engineering and Electronics, Academy of Sciences, Czech Republic. Since 1997, he has been with the University of Southampton, U.K., where he is currently a Professor of wireless communications with the School of Electronics and Computer Science. His research interests include wireless communications, wireless networks, signal processing for wireless communications, molecular communications, and nano-networks. He has published over 350 research papers in journals and conference proceedings, has authored/co-authored three books, and has also published several book chapters. He is a Fellow of the IET, U.K., and a Distinguished Lecturer of the IEEE Vehicular Technology Society. $\mathrm{He}$ has served as an associate editor for several academic journals, has coorganized several special issues, and has acted in different roles for conference organization.

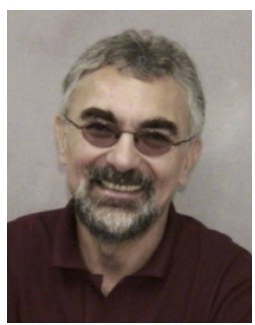

Lajos Hanzo (M'91-SM'92-F'04)

FREng, FIEEE, FIET, Fellow of EURASIP, DSc received his degree in electronics in 1976 and his doctorate in 1983. In 2009 he was awarded an honorary doctorate by the Technical University of Budapest and in 2015 by the University of Edinburgh. In 2016 he was admitted to the Hungarian Academy of Science. During his 40 -year career in telecommunications he has held various research and academic posts in Hungary, Germany and the UK. Since 1986 he has been with the School of Electronics and Computer Science, University of Southampton, UK, where he holds the chair in telecommunications. He has successfully supervised $112 \mathrm{PhD}$ students, co-authored 18 John Wiley/IEEE Press books on mobile radio communications totalling in excess of 10000 pages, published 1776 research contributions at IEEE Xplore, acted both as TPC and General Chair of IEEE conferences, presented keynote lectures and has been awarded a number of distinctions. Currently he is directing an academic research team, working on a range of research projects in the field of wireless multimedia communications sponsored by industry, the Engineering and Physical Sciences Research Council (EPSRC) UK, the European Research Council's Advanced Fellow Grant and the Royal Society's Wolfson Research Merit Award. He is an enthusiastic supporter of industrial and academic liaison and he offers a range of industrial courses. He is also a Governor of the IEEE ComSoc and VTS. During 2008 - 2012 he was the Editor-in-Chief of the IEEE Press and a Chaired Professor also at Tsinghua University, Beijing. For further information on research in progress and associated publications please refer to http://www-mobile.ecs.soton.ac.uk 